\title{
Electrodeposited Biocoatings, Their Properties and Fabrication Technologies: A Review
}

\author{
Andrzej Zielinski *(D) and Michal Bartmanski \\ Department of Materials Engineering and Bonding, Gdansk University of Technology, Narutowicza 11/12, \\ 80-233 Gdansk, Poland; micbartm@pg.edu.pl \\ * Correspondence: azielins@pg.edu.pl; Tel.: +48-501-329-368
}

Received: 27 July 2020; Accepted: 9 August 2020; Published: 12 August 2020

\begin{abstract}
Coatings deposited under an electric field are applied for the surface modification of biomaterials. This review is aimed to characterize the state-of-art in this area with an emphasis on the advantages and disadvantages of used methods, process determinants, and properties of coatings. Over 170 articles, published mainly during the last ten years, were chosen, and reviewed as the most representative. The most recent developments of metallic, ceramic, polymer, and composite electrodeposited coatings are described focusing on their microstructure and properties. The direct cathodic electrodeposition, pulse cathodic deposition, electrophoretic deposition, plasma electrochemical oxidation in electrolytes rich in phosphates and calcium ions, electro-spark, and electro-discharge methods are characterized. The effects of electrolyte composition, potential and current, $\mathrm{pH}$, and temperature are discussed. The review demonstrates that the most popular are direct and pulse cathodic electrodeposition and electrophoretic deposition. The research is mainly aimed to introduce new coatings rather than to investigate the effects of process parameters on the properties of deposits. So far tests aim to enhance bioactivity, mechanical strength and adhesion, antibacterial efficiency, and to a lesser extent the corrosion resistance.
\end{abstract}

Keywords: coatings; electrocathodic deposition; electrophoretic deposition; plasma electrochemical oxidation; electro-spark deposition; electro-discharge deposition; bioactivity; antibacterial efficiency; mechanical strength; corrosion resistance

\section{Introduction}

The coatings are widely applied for different purposes in the national economies and households. The metallic and polymeric coatings as paints and lacquers are used for the protection of metallic ferrous and nonferrous constructions, in buildings and houses for long term anti-corrosion protection and esthetic effects. The hard coatings are applied to increase the wear resistance. Many functional coatings have been so far proposed and developed. Just recently, for example, gas-barrier thin film coatings for food and beverages [1], the coatings for automotive brake discs [2], tribological coatings [3], functional textile coatings [4], super-hydrophobic anticorrosion coatings [5], and cermet coatings for erosion-corrosion-protection [6] were investigated.

The various coatings have been applied for many years in medicine to improve the properties of the interface between implants and tissues, which determine the biocompatibility and healing time. In past years, the reviews on such functional coatings for dental implantology [7], biocompatible coatings for bone implants [8], ion substituted hydroxyapatite thin films [9], titanium implants polymeric coatings [10], ceramic coatings for osteoporotic bones [11], and pulse laser deposited animal-originated calcium coatings [12] can be found.

The coatings for biological applications, called biocoatings, may be made of metals, polymers, ceramics, and bioglasses, or can be composite coatings, co-deposited, or formed layer-by-layer (hybrid or 
sandwich coatings). They may be formed by various techniques such as direct electrocathodic deposition (ECD), pulse electrocathodic deposition (PED), electrophoretic deposition (EPD), plasma electrochemical oxidation (PEO) called also micro-arc oxidation (MAO) in calcium, and phosphorus-containing phosphate solutions, chemical vapor deposition (CVD), plasma vapor deposition (PVD), magnetron sputtering, pulsed laser deposition, and many others. They may coat the solid or porous substrates. The biocoatings obtained by electrodeposition are included in many reviews and it is interesting to look for the change of the most popular fabrication techniques from the past till now. Some time ago [13], typical coating methodologies were the ion beam assisted deposition, plasma spray deposition, pulsed laser physical vapor deposition, magnetron sputtering, sol-gel derived coatings, electrodeposition, micro-arc oxidation, and laser deposition. In [14], described methods such as electrodeposition, electrografting, micro-arc deposition, electropolymerization, and electrophoretic deposition of polymers, metals, metal oxides, and ceramics on surfaces of titanium, stainless steels, magnesium alloys, and cobalt alloys were described In another paper [15], the review on surface treatment of titanium alloys focused on anodization mentioning the plasma electrochemical oxidation (PEO) method. In [16], the sol-gel and electrochemical deposition methods were described for many covered metals. The excellent characterization of calcium phosphates, including their coatings, was given in [17]. The achievements in the deposition of Ca-P coatings by sol-gel, thermal spray, magnetron sputtering, electrophoretic deposition, and micro-arc oxidation were recently reviewed in [18]. Liu et al. [19] exhaustively described the PEO technique, plasma spraying, ion implantation, laser surface treatment, sol-gel method, and friction stir processing for biological applications. The review on surface biofunctionalization on implantable metals by Ca-P coatings obtained in various ways, including electrodeposition, was presented in [20]. For popular in recent years Mg alloys applied for biodegradable implants, the cathodic electrodeposition in [21], and the electrophoretic method in [22] was characterized.

This paper is aimed to critically review the most recent, in significant part, achievements in two fields. In the first part of the review, the different coatings deposited by any electrodeposition method (EDM) are described based on their material composition, i.e., metallic, ceramic, polymer, and composite coatings, with attention paid to the effects of component(s) on the coating properties and its application. The further section shows several, most popular deposition technologies applied for biocoatings, and the process parameters, focusing on their effects on properties of coatings. In particular, the deposition techniques here described are those performed in electrolytes and under electric field, limited to ECD, EPD, PEO, electro-spark deposition (ESD), electro-discharge deposition (EDP), and electropolymerization (EP). Thus, the material effects followed by processing effects are subsequently presented together with, at the end of each of the sections, a short summary of the state-of-the-art and prognosis concerning future research.

\section{Biocoatings}

\subsection{Metallic Coatings}

The electrodeposited metallic coatings for medical purposes are rare. In [23], the bismuth nanowires were deposited on glassy carbon substrates by the ECD method for electro-reduction of folic acid and its quantitative determination, constituting in such a way a biosensor. In another report [24], a new electrodeposited platinum-iridium coating on platinum, as an alternative to the iridium oxide, was developed for implanted microelectrode arrays being the essential tools in the field of neural engineering. As to increase the corrosion resistance and improve the hydroxyapatite (HAp) deposition, the tantalum was put on the titanium oxide film and then modified with organophosphonic acids [25]. The strontium coating was obtained in [26], demonstrating its positive effect on cell differentiation. In [19], the graphitic carbon nitride nanosheets were used for the self-templated electrodeposition of copper and copper oxide nanostructures for the detection of glucose. In other work [27], the electrodeposition of gold nanowires performed under potentiostatic control, designed as 
biosensors, was carried out. Finally, the nickel nanoparticles were electrodeposited on $\mathrm{TiO}_{2}$ nanotubes by either direct current (DC) or cyclic voltammetry (CV) methods to obtain the ferromagnetic coatings [28].

\subsection{Ceramic Coatings}

\subsubsection{Phosphate Coatings}

The calcium phosphates (Ca-P), in particular, hydroxyapatite (HAp) and nanohydroxyapatite (nanoHAp), are widely applied in implantology [17] as bone substitutes or bioactive coatings accelerating the primary fixation time of implants. The healing time and the proper adhesion of the coating to the substrate, even under imposed mechanical stresses, at simultaneous lack of adverse effects to the tissues, resulted in an enormous development of HAp bone substitutes and, later on, nanoHAp coatings. The HAp coatings have been developed for the enhancement of bioactivity on titanium implants, and the high number of earlier papers has been focused on the optimization of coatings. Despite that, the characteristic feature of recent research is the use of other than titanium, metallic, and non-metallic surfaces.

In the past, the Ca-P phosphates, mainly stoichiometric or non-stoichiometric hydroxyapatites, were developed as coatings mostly for titanium and its alloys by an application of a direct voltage or current control. For example, in [29] the evolution of the first stages of the crystallization of an electrochemically deposited calcium carbonate was investigated. During the growth of the initial nuclei, the surface of the electrode was covered progressively by the growth of flat multilayers having triangular faces, ranged from one to several molecular layers of calcium carbonate.

The bioactivity is an essential feature of Ca-P coatings. In an early work [30] the electrodeposited calcium phosphate coatings were transformed into apatites during immersion in the simulated body fluid (SBF) at $36.5^{\circ} \mathrm{C}$ for 5 days what is still assumed to be a confirmation of the above property.

Recently, the different methods were applied to improve the properties of coatings and their adhesion to solid titanium and its alloys, associated often by pre-treatment of post-treatment. The chemical treatment with $\mathrm{H}_{2} \mathrm{O}_{2}$ before PED was also often used, as in research [31], in which the porous coating comprising of HAp was formed on the treated titanium surface. The coating was transformed into carbonate- and calcium-deficient HAp layers with a bonelike crystallinity during further immersion in the SBF. To increase adhesion, Ca-P coatings were obtained on pre-calcified titanium, demonstrating a presence of the $\mathrm{TiO}_{x}$ layer with calcium and hydroxyl groups on the surface after this chemical treatment and resulting in a lower water contact angle and lower surface energy than on unmodified titanium surfaces [32]. As concerns the post-treatment, in [33], titanium was firstly subjected to very short electrodeposition and then post-treated in $\mathrm{NaOH}$ solution to improve the adhesion. Adhesion was also observed to improve [34] with the increasing electrodeposition time accompanied by a change in the surface morphology from smooth to plate-like, featuring elongated plates, ribbon-like, and finally sharp needle structures. The adhesion of HAp coating on metallic biomaterials is a crucial parameter, dependent on the coating type, deposition method, and substrate [35]. Among four standard techniques of deposition, namely sol-gel, dip coating, electrochemical deposition, and thermal spraying, adhesion of electrodeposited coatings was reported as the lowest. Such a conclusion explains the current trend to optimize this significant feature of biocoatings.

Several studies on Ca-P coatings on titanium biomaterials were performed in the last period with pulse electrodeposition (PED). For titanium irradiated with a high energy electron beam and subject to PED, the lotus flower-like morphology was observed after deposition in SBF in the presence of $\mathrm{H}_{2} \mathrm{O}_{2}$ [36]. In [37], in the presence of $\mathrm{H}_{2} \mathrm{O}_{2}$, the coatings composed of HAp and tricalcium phosphate (TCP) were synthesized by PED, revealing the important dissolution-precipitation characteristics and enhanced corrosion protection properties. In another work [38], the application of ultrasonic waves instead of magnetic stirring during PED resulted in the uniform and refined size of plate-like Ca-P crystals. 
The HAp and nanoHAp coatings were deposited by EPD on different substrates, for example on NiTi [39], Ti [40-42], oxidized Ti [43-46], and Ag [47]. The Mg-containing hydroxyapatite coatings were also obtained on Ti alloy by MEO with destination for dental materials [48]. For the same purpose, in [49], the HAp nanoparticles were formed on Ti-Nb-Zr alloys by PED followed previous oxidation to nanotubular oxide surface layer. The simultaneous precipitation and electrodeposition of HAp were claimed to be useful for dentistry and orthopedic applications [50].

The HAp coatings can be formed on porous surfaces (scaffolds). After deposition [51] of the Ca-P coatings on the 3D surface of Ti scaffolds made by selective laser melting, the coating morphology was found to depend on the distance from the surface. The top and the bottom surfaces of SLM-Ti scaffolds exhibited continuous and instantaneous nucleation, but the EPD processes at different depth of SLM-Ti scaffolds did not follow the surface processes because of the non-uniform distribution of the potential and the current inside porous structures.

As a treatment positively influencing adhesion, the presence of a nanotubular oxide layer on titanium was extensively applied. For example, the nanotubular surface with the tube length of $560 \mathrm{~nm}$ coated with HAp showed higher cell density, higher live cells, and more spreading of MC3T3-E1 cells than that growing on titanium plate surface [52].

The use of HAp-based composite coatings with antibacterial properties is an important research direction. In [53], the calcium phosphate coatings were prepared and then loaded with an antiseptic agent, chlorhexidine digluconate, by a single-step co-deposition. The coating was effective against Staphylococcus aureus and Escherichia. coli bacteria strains.

Among other metallic biomaterials, the stainless steels were the object of some investigations. In [54], the HAp coating was deposited on 316L stainless steel (SS) in the presence of a significant amount of $\mathrm{H}_{2} \mathrm{O}_{2}$ by both the direct and pulsed current electrodeposition methods. In [55], the effects of temperature and $\mathrm{H}_{2} \mathrm{O}_{2}$ content on the morphology, structure, and composition of the coating were confirmed. Surprisingly, in [56], the HAp coatings plated by ECD on 316L steel, but also Ti and Ti6Al4V alloy, increased corrosion rate, likely because of highly porous coating and crevice corrosion. The PED seems a more efficient method as shown in [57], in which the perfect coatings of biocompatible HAp were obtained at various current densities over the SS316 steel. In later research [58], the phosphate coatings, transformed to hydroxyapatite, had thickness from $300 \mathrm{~nm}$ up to $2 \mu \mathrm{m}$, depending on the deposition mode (continuous or pulsed reverse), and voltage or current parameters, and microstructure of the flake type demonstrating the spontaneous passivation under anodic polarization, and the corrosion potential improved with the increasing presence of hydroxyapatite phase in the coating. An interesting process to form bi-layer (hybrid) coatings [59], the nanoHAp and phosphorus-rich electroless nickel composite coating as an interlayer, was developed before electrodeposition of pure HA coating on stainless steel.

Another group of biometals deposited with HAp was CoCrMo alloys. In [60], the Ca-P coatings were obtained consisting of fine crystallized HAp. On the other hand, for such coatings [61], the coating was weakly hydrophilic, with the phase angle over $85^{\circ}$.

The next group of biometals deposited with HAp coatings was Ni-Ti alloys. In earlier work [62], such coatings were developed to improve the cardiovascular stents with less restenosis than drug-eluting stents. In another study [63], a bipolar pulsed current was used for the electrochemical deposition resulting in the crystalline coating composed of pure HAp nanowalls. Thorough studies of microstructure [64] showed for PED of Ca-P coatings that plate-like and needle-like morphologies were formed in dilute and concentrated solutions, respectively, and HAp appeared at increasing the pulse current density. The coatings obtained in dilute solution showed the best biocompatibility, the highest cell density, and cell proliferation, explained by the stability of the plate-like coating in biological environments. In [65], the surface modification of the NiTi alloy was accomplished before deposition by anodic oxidation and subsequent heat treatment.

Magnesium alloys have become promising materials in the medical field, particularly in tissue engineering applications. In [66], the HAp coating, electrodeposited on AZ91D magnesium alloy and 
comprising of brushite, lowered the biodegradation rate of $\mathrm{Mg}$ alloy in SBF, being the main aim of use of such coatings. The deposited coatings on Mg-1Ca alloy demonstrated improved corrosion resistance in Hank's SBF [67]. In another work [68], three kinds of Ca-P coatings, brushite, HAp, and the most stable and corrosion-resistant fluoridated hydroxyapatite (FHAp) were fabricated by electrodeposition on a biodegradable $\mathrm{Mg}-\mathrm{Zn}$ alloy. To effectively control the degradation of magnesium alloys, a uniform nano-hydroxyapatite (nanoHAp) coating was applied on AZ31 magnesium alloy using both direct and pulse voltage electrodeposition methods [69]. In similar research on nanoHAp coatings [70] obtained by PED on the porous Mg-2 wt.\% Zn scaffold, the corrosion current density reduced from 8 to $0.15 \mathrm{~mA} / \mathrm{cm}^{2}$. The next study on nanoHAp deposition [71] on $\mathrm{Mg}$-2Zn scaffolds performed by PED and post-treatment with alkaline solution demonstrated better biodegradation behavior and biocompatibility, and adherence and proliferation of the MG63 cells when compared to an uncoated alloy. In a more recent report [72], four electrodeposition methods, i.e., the constant potential method, the pulsed potential method, the constant current method, and the current pulsed method, were evaluated. Using the constant current and the current pulsed techniques, smooth uniform coatings were obtained onto AZ31 alloy for both low and high-frequency pulse. Finally, in [73], the Ca-P coatings on Mg implants were subjected to hydrothermal treatment, anodization, and plasma electrolytic oxidation.

The HAp coatings were seldom electrodeposited on ceramics. In [74], HAp coatings, from 1 to $10 \mu \mathrm{m}$ in thickness, were prepared on human enamel by electrodeposition. They exhibited an acicular morphology and had a tight contact with the substrate.

The HAp coatings were sometimes deposited on polymer surfaces and scaffolds. By the templateassisted PED, the calcium-deficient polycrystalline HAp nanowires and nanotubes were obtained, nucleating preferentially on gold nanoparticles polycarbonate membrane, as nanowires on smaller Au particles and as nanotubes on $400 \mathrm{~nm}$ nanoparticles [75]. HAp, carbonate apatite, and dicalcium phosphate dihydrate (brushite) were deposited on carbon/carbon (C/C) composites [76]. A uniform and dense nanoHAp coating with the nanorod-shaped structure were fabricated on carbon nanotubes (CNTs), applied to reinforce the coating [77]. The ultrasonic-assisted electrodeposition was attempted to prepare uniform and smooth hydroxyapatite HAp coating on the surface of the PLA/PVA filaments, which can be potentially applied as biodegradable bone scaffolds [78]. The crystal's growth stages of HAp went through spherical particles, plate-like, and needle-like crystals when electrodeposited from 15 to $60 \mathrm{~min}$.

\subsubsection{Substituted Phosphate Coatings}

The substituted hydroxyapatites include fluoridated hydroxyapatite (FHAp) and some other inorganic ions substituting calcium ions. The main reason for such design is an expectation of antibacterial effects and better bioactivity. Different substrates and electrodeposition techniques were applied.

As concerns, the titanium alloys, the $\mathrm{Sr}, \mathrm{Mg}$, and $\mathrm{Zn}$ substituted hydroxyapatite coatings, achieved by PED, exhibited excellent corrosion resistance on Ti6Al4V alloy, in particular at the prolonged pulse off time [79] and strontium-substituted calcium phosphate coatings on Ti6Al4V alloy demonstrated the best osteoblast cells activity and the osteoclast cells proliferation at 5\% Sr [80]. In [81], magnesium-doped HAp (MgHAp) was obtained on titanium with a previously formed nanotubular oxide layer, showing only slightly increasing the bond strength. For the strontium/copper substituted hydroxyapatite (SrCuHAp) coatings on titanium, $\mathrm{Cu}$ improved its antimicrobial properties, and $\mathrm{Sr}$ enhanced the biocompatibility [82]. In other work [83], strontium and manganese co-substituted hydroxyapatite (SrMnHA) crack-free and dense coatings on titanium were prepared by ECD resulting in the decrease in the corrosion current density, strongly hydrophilic surface and better cell morphology, adhesion, spreading, and proliferation, and expression of alkaline phosphatase (ALP) better than on HAp. In similar research [84], the presence of strontium in nanostructured SrHAp coating deposited by ECD was shown again to positively influence the behavior of cells on a titanium surface, i.e., cell viability, adhesion, cell morphology, and the cytoskeletal structure of bone marrow mesenchymal 
stem cells (MSCs). For the hydroxyapatite doped with silver and manganese (AgMnHAp), deposited on $\mathrm{TiO}_{2}$ nanotubes [85], corrosion resistance decreased by almost two orders of magnitude, and an excellent antimicrobial efficacy was observed with a 100\% reduction in viable cells. The lanthanum and copper substituted hydroxyapatite ( $\mathrm{La} / \mathrm{Cu}-\mathrm{HAP}$ ) coatings made on titanium by ECD demonstrated excellent biocompatibility and bioactivity [86]. The cobalt-substituted Ca-P coatings on Ti22Nb6Zr alloy [87], protected the surface against corrosion in the physiological environment and promoted the development of the cells. The most recently [88], the Ca-P coatings were developed by pulse current deposition onto Ti6Al4V alloy, and after doped with $\mathrm{Zn}^{2+}, \mathrm{Mg}^{2+}, \mathrm{Sr}^{2+}$, and $\mathrm{Ag}^{+}$ions showed better biocompatibility, but also lowered corrosion resistance compared to no-doped Ca-P coating or bare metal.

In the case of steels as substrates, the deposition of $\left(\mathrm{Zn}^{2+}, \mathrm{Cu}^{2+}\right.$, and $\left.\mathrm{Ag}^{+}\right)$substituted FHAp coatings were obtained on the $316 \mathrm{~L}$ steel by ECD to further enhance the antibacterial efficiency of FHAp [5].

For Mg alloys [89], PED in the presence of $\mathrm{H}_{2} \mathrm{O}_{2}$ was applied to improve the corrosion resistance and bioactivity of (nano)FHAP coating on $\mathrm{Mg}-\mathrm{Zn}-\mathrm{Ca}$ alloy. A silicon doped calcium phosphate coating was obtained [90] on AZ31 alloy by pulse electrodeposition demonstrating slow degradation rate in SBF, an enhanced corrosion resistance, good cell growth, and enhanced cell proliferation of MG63 osteoblast-like cells, as well as increased activity of ALP. The magnesium-substituted coatings had excellent biocompatibility and no adverse effect. Recently [91], a Si-HAp coating was achieved by PED on an $\mathrm{Mg}-\mathrm{Zn}-\mathrm{Ca}$ alloy to improve biocompatibility, bioactivity, and osteoconductivity.

The substituted HAp coatings were also deposited on polymer substrates. Strontium and magnesium substituted Ca-P coatings were formed on C/C composites by PED, showing the flake-like morphology with a dense and uniform structure that could induce the formation of apatite layers and decrease the corrosion rate of the $\mathrm{C} / \mathrm{C}$ composites in simulated body fluid [92]. In other research [93], nanocrystalline zinc HAp was prepared by ECD on a titanium-coated silicone provided bioactive properties based on the fibroblast ingrowth and limiting the number of viable E. coli.

\subsubsection{Oxides' Coatings}

The metal oxides for biological applications were seldom deposited. In [94], PED was applied to co-deposit iridium oxide and human plasma proteins. In [95], a novel electrochemical sensor of creatinine was constructed on the glassy carbon electrode. The graphene oxide (GO) and dopamine were transformed to polydopamine and reduced graphene oxide (rGO) film and finally decorated by PED with the nanoparticles $\mathrm{Cu}$ by cyclic voltammetry.

\subsection{Polymer Coatings}

The most applied polymer for electrodeposited biocoatings was chitosan. In [96], the chitosan layer was deposited by ECD on a previously formed porous $\mathrm{TiO}_{2}$ film layer. It is noteworthy that there was an exciting attempt [97] of electrodeposition of bioabsorbable polymer on NP-eluting stent made of stainless steel. Chitosan-mussel protein coatings were proposed to decrease the biodegradation of $\mathrm{Mg}$ in body fluids [98]. Photo cross-linking copolymer based on methacrylates deposited by EPD decreased the release of nickel ions from NiTi reducing cytotoxicity inducing by nickel [99]. The polyether ether ketone (PEEK) coatings [100] demonstrated excellent wear resistance, more than two hundred times greater compared with that of the uncoated Ti-13Nb-13Zr alloy.

\subsection{Composite Coatings}

\subsubsection{Ceramics-Ceramics Coatings}

The highest group of such coatings is based on hydroxyapatite-phosphate composition with often some ceramics. In [101], a coating composed of HAp and Ca-P phosphate was synthesized over 316L stainless steel through PED showing an increased corrosion resistance and good bioactivity. HAp 
and $\mathrm{HAp} / \mathrm{ZrO}_{2}$ composite coatings were [102] electrodeposited on 316L stainless steel to improve electrochemical behavior and bonding strength. When adding $\mathrm{ZrO}_{2}$ at a concentration of $10 \mathrm{~g} / \mathrm{L}$, the corrosion resistance was improved 30 times, and bonding strength between the coating and the substrate increased from 11.6 MPa in pure HAp to 20.8 MPa in composite coatings. Another approach [103] was a deposition of a composite coating comprised of HAp, Ca-P, and multi-walled carbon nanotubes (MWCNTs) on SS316 steel surface by PED showing increased the overall coating modulus of elasticity in the range of 6-10 GPa, a uniform and fast spreading of cells over the coating surfaces and five times higher corrosion resistance.

The Hap-silicate coatings were also developed. In [104], such porous composite coating was obtained demonstrating higher bond strength and better corrosion resistance, and enhanced proliferation of MC3T3-E1 osteoblast cells grown on the $\mathrm{HAp} / \mathrm{CaSiO}_{3}$ coating compared to pure HAp. More complex [105] the electroplated zinc-doped hydroxyapatite-silicate coatings presented porous structure, higher corrosion resistance than bare Ti, and improved cell morphology, adhesion, spreading the proliferation of the MC3T3-E1 cells, and expression of ALP alkaline phosphatase comparing to HAp coating.

The nanocomposite coating comprising of calcium titanate, sodium titanate nanotubes, and rutile was prepared on a titanium substrate by a combination of ECD and chemical post-treatment [106] resulting in similar adhesion strength and increasing hardness and corrosion resistance were increased.

The zinc-halloysite nanotubes/( $\left.\mathrm{Sr}^{2+}, \mathrm{Sm}^{2+}\right)$ substituted HAp hybrid coating was obtained on Ti6Al4V alloy by the ECD. The enhanced corrosion resistance due to Zn nanotubular layer, significant in-vitro antibacterial efficiency and cell viability studies, and anti-corrosion stability in SBF was observed [107,108].

The coatings composed of HAp and graphene oxide (GO) $[109,110]$ were deposited by ultrasoundassisted PED on the anodized heat-treated surface of the titanium bringing out the highest nano-hardness (3.08 GPa) and Young's modulus (41.26 GPa). Similar hybrid coating composed of the GO inner layer and $\mathrm{GO} / \mathrm{MgHAp}$ outer layer was prepared on carbon/carbon composites [111] with the bonding strength between the coating and carbon composite over $80 \%$ higher than without the oxide.

Carbon nanotubes (CNTs) were also proposed as material for brittle HAp. In earlier research [112], the CNTs reinforced HAp on the Ti substrate demonstrated high nanohardness, enhanced corrosion resistance, and cell viability. In more recent work [113], the addition of CNTs increased nanohardness over $52 \%$ and Young's modulus over $41 \%$, and $16 \%$ in the adhesion strength thanks to improving integrity, crystallinity, and decreasing Young's modulus mismatch of this coating with titanium substrate. Such coatings were also prepared on AZ31 magnesium alloy by ECD and EPD [114], resulting in decreasing seriously corrosion density from 44.2 to $0.7 \mu \mathrm{A} / \mathrm{cm}^{2}$. In [115], different carbon nanostructures, namely exfoliated graphene, RGO, and MWCNTs were co-deposited along with in-situ formed HAp-Ca-P phases. The MWCNTs were deposited together with HAP also by the EPD technique $[116,117]$.

Some oxides were applied for composite HAp coatings. In [118], $\mathrm{HAp}-\mathrm{ZrO}_{2}-\mathrm{TiO}_{2}$ nanocomposite coatings were synthesized with new morphologies of electrodeposited HAp, such as micro-nanorods. The corrosion current density of coatings was equal to $0.01 \mu \mathrm{A} / \mathrm{cm}^{2}$ while the bare substrate was $1.5 \mu \mathrm{A} / \mathrm{cm}^{2}$, and porosity was decreased from $46 \%$ for HAp coating to $6 \%$ for composite coating. A $\mathrm{ZnO}$ nanoprism/Zn substituted HAp hybrid layer was prepared on carbon fibers by a two-step electrodeposition method [119].

The HAP was also deposited with borium nitride (BN) by EPD [120] and bioglass and HAp [121].

\subsubsection{Ceramics-Polymer Coatings}

A novel implant coating material containing graphene oxide and collagen, and HAp was fabricated on Ti16 Nb electrooxidized alloy [122] with increased corrosion resistance, superhydrophilic property, antibacterial resistance, and adhesion strength. In other research [123], a novel poly(3,4-ethylene dioxythiophene) based nanocomposite coatings with different contents of FHAp nanoparticles on 
a Ti-Nb-Zr alloy were developed demonstrating enhanced hardness, a contact angle, and corrosion protection than the pure polymer coatings. The fabricated nanocomposite coating supported the cell adsorption and proliferation of MG-63 cells and showed antibacterial performance. Another research showed [124] for a composite coating of HAp-polypyrrole, synthesized by PED over stainless steel, the increased corrosion resistance by ten-fold as compared to bare polymer coating and supported the growth of MG63 cells. Finally, in [94], a composite coating of iridium oxide and human plasma proteins was obtained by PED.

Some antibacterial nanocomposite coatings were developed [125] as the HAp-Ag-chitosan monolayers or multilayers containing HAp-chitosan and Ag-chitosan layers, with a thickness in the range of $0-20 \mu \mathrm{m}$. As assumed, the $\mathrm{Ag}^{+}$release rate from the Ag-chitosan layer can be reduced in the layered structure using a HAp-chitosan layer. The multilayer coatings provided corrosion protection of the stainless steel in physiological solutions. Similar biocomposite coating containing chitosan, silver, and hydroxyapatite was developed on anodized titanium substrate by ECD [126] - 6- $\mu \mathrm{m}$ thick. The exhibited antibacterial activity against Gram-positive and Gram-negative bacterial strains was due to the synergistic effect of silver and chitosan, with no toxicity concerning MC3T3-E1 cells.

The electrodeposition of a chitosan/layered double MgAl hydroxides was applied to obtain hydro-membranes for protein release triggered by an electrical signal [127]. Chitosan was also co-deposited with HAp, ZNHAp, and MWCNTS [128].

Another approach based on HAp was attempted by the addition of the poly-l-lysine (PLL) + 3,4-dihydroxy benzyl aldehyde (DHBA) and $\mathrm{TiO}_{2}$ [129] or PEEK [130]. The octadecyltrichlorosilane layer on HAp coatings was shown to be effective in preventing the acid corrosion of dental samples [131].

\subsubsection{Metal-Ceramics Coatings}

The nickel-MWCNTs coatings were co-electrodeposited [132]. The chemically shortened nanotubes were behaved as inert particles and embedded into the nickel matrix, while the long functionalized nanotubes showed metallic behavior, and during electrodeposition, they were incorporated into the nickel matrix. The hydroxyapatite-zinc coating was attained [133] using ECD possessing excellent adhesion to the substrate. In [134], a two-stage electrochemical synthesis method was used to prepare antimicrobial Ag-HAp composite coatings. In the first stage, a titanium substrate was coated with HAp by ECD, and in the second stage, silver nanoparticles were deposited onto the HAp layer through electrochemical reduction of aqueous $\mathrm{Ag}^{+}$to $\mathrm{Ag}^{0}$. Similar research was described by Bartmanski et al. for nanoAg [135,136] and nanoCu [137]. The coating composed of nanoHAp, MWCNTs, nanoAg, and nanoCu were developed to obtain antibacterial efficiency, high adhesion, and mechanical strength of coatings [138] also with additions of nanoAg and nanoCu. The ultra-fine Pd-Ag-HAp nanoparticles electrodeposited on protruded $\mathrm{TiO}_{2}$ layer [139] were considered as plausible for dental implants as increasing their biocompatibility.

The influence of the silver nanoparticle (AgNPs) concentration in solution on the electrodeposition of $\mathrm{Zn}-\mathrm{Cu} / \mathrm{AgNP}$ composite coatings was studied by PED [140]. Antimicrobial tests revealed $95 \%-100 \%$ inhibition of bacterial growth after 10 min of contact for the $\mathrm{Zn}-\mathrm{Cu} / \mathrm{AgNP}$ coating with an AgNPs content of 0.28 wt.\% against the E. coli.

\subsubsection{Metal-Polymer Coatings}

In [141], composite coatings consisting of polypyrrole (PPy) and $\mathrm{Nb}_{2} \mathrm{O}_{5}$ nanoparticles were obtained by ECD on 316L stainless steel resulting in enhanced hardness, superior biocompatibility and enhanced corrosion protection as compared to pure PPy coatings. Other research [142] developed the polyethyleneimine film decorated with gold nanoparticles, electrodeposited on glassy carbon electrode, and forming the platform adequate to immobilize proteins for applications as a tool in biotechnology. 


\subsubsection{Polymer-Polymer Coatings}

In [143], the alginate/chitosan layer-by-layer composite coatings were prepared on titanium substrates. No cytotoxicity was observed, and the alginate coating was more beneficial for cells' growth than chitosan coating. More recently [144], bioactive composite coatings based on polypyrrole (PPy)/chitosan were obtained by in situ electrochemical polymerizations with enhanced surface hydrophilicity and enhanced protective performance compared to pure PPy. Recently, partly biodegradable coating composed of chitosan and Eudragit (copolymer of methacrylic acid used for controlling drug release) was developed [145]. The gallium-modified chitosan/poly(acrylic acid) bilayer was proposed for titanium dental implants to prevent their failures [146]. Polymerized microemulsions of PMMA and soy lecithin on 316L stainless steel were also elaborated [147].

\subsection{Effects of Component(s) on Properties of Biocoatings}

The coatings used or developed for medical purposes can be made of different materials. That is determined by existing specific needs or, more often, by a demand to increase biocompatibility and bioactivity, and implement antibacterial efficiency. Therefore, the coatings are in a great number designed for metallic implants made of stainless steels, titanium, NiTi alloys.

The one-component metallic coatings were proposed for particular applications, mainly for construction of biosensors and, only in single cases, for others such as the creation of ferromagnetic coatings or as additional layers. This main application is related to the dependence of electrochemical potential of metals (half-cells) on the environmental signals like $\mathrm{pH}$, temperature, and oxygen or hydrogen amount. The use of some oxides as single layers was scarce.

The ceramic coatings, in the past, were developed as simple phosphates (Ca-P: triphosphate (TCP), octacalcium phosphate (OCP), hydroxyapatite (HAp), and others). However, such coatings, even cheap and of good bioactivity, were prone to brittle cracking, often weakly adjacent to the base, and did not prevent the development of biofilm. Four solutions were then proposed: to modify the chemical formula of phosphate, to substitute phosphates by other substances, to create the composite HAp-based coatings, or to look for the best deposition technology and process parameters (as discussed in next section).

Among substituted phosphates, the fluorohydroxyapatite in which a fluoro group substituted a hydroxyl group, was often investigated. Besides, the hydrogen atoms were substituted by several metallic elements such as $\mathrm{Zn}, \mathrm{Sr}, \mathrm{Cu}, \mathrm{Ag}$, and $\mathrm{Mn}$, mainly for titanium made implants. The attempts to decrease the solubility rate of biodegradable $\mathrm{Mg}$ alloys for implants by the development of SiHAp should also be noted.

Among one-component polymeric coatings, they were used for different purposes: for example, chitosan for increasing the bioactivity or to decrease the $\mathrm{Mg}$ degradation, methacrylates for prevention of Ni release from NiTi alloys, and PEEK as an anti-wear coating for biopairs.

The one-component coatings are usually relatively cheap, but their applications are limited as the obtained surface properties are not fully satisfactory. Therefore, the two or three or even four-component coatings were extensively developed.

The mostly applied are coatings composed of two ceramics such as HAp (or, more and more often, nanoHAp), silicates, oxides (mainly reduced graphene oxide rGO), CNTs (mainly MWCNTs), sometimes other substances as $\mathrm{BN}$ or halloysite. These coatings often demonstrated enhanced bioactivity and hardness, but their resistance to cracking, adhesion, and antibacterial action could be acknowledged as satisfactory only in part.

The promising direction is developing the metal-polymer coatings, which are usually based on HA with additions of chitosan, collagen, polypyrrole (Py), and others, sometimes implemented also with oxides. Such coatings may be well adjacent to the surface, stronger compared to pure ceramic coatings, and possess better biological properties. This research direction seems not so far developed, but very promising. 
Other composite coatings were less investigated. It noteworthy that the metal-ceramics coatings based on nanoHAp implemented with antibacterial nanometals $\mathrm{Ag}$ and $\mathrm{Cu}$ were used for orthopedic and dental implants. Among the polymer-polymer coatings it is worth noting the appearance of several biodegradable or protective coatings.

Summarizing, there are two most promising development directions of research coatings. The first scientific goal is to create the coating highly bioactive, biocompatible, of high adhesion to both orthopedic and dental implants, but also resistant to corrosion and mechanical stresses, and antibacterial. Such coatings could be based on (substituted or no) nanoHAp and may include several other components: polymer for better crack resistance and additional biological properties (chitosan mainly recommended as a cheap and efficient component), nanoAg (or AgHAp, SrAgHAp, ZnAgHAp, and MnAgHAp). The second objective, less developed even if very promising, is to create the coatings with some specific functions as smart coatings for biosensors and long-term antibacterial protection, thin multifunctional coatings for stents, and wear-resistant coatings. The effect of used material on coating properties and possible applications is then highly significant, and the influence of applied technology, as demonstrated in other sections, is sometimes important and in other cases rather minor.

Figure 1 demonstrates the electrodeposition method made (EDM) biocoatings. They have been divided at first according to whether they are on or multicomponent coatings, and then they have been listed based on the main materials.

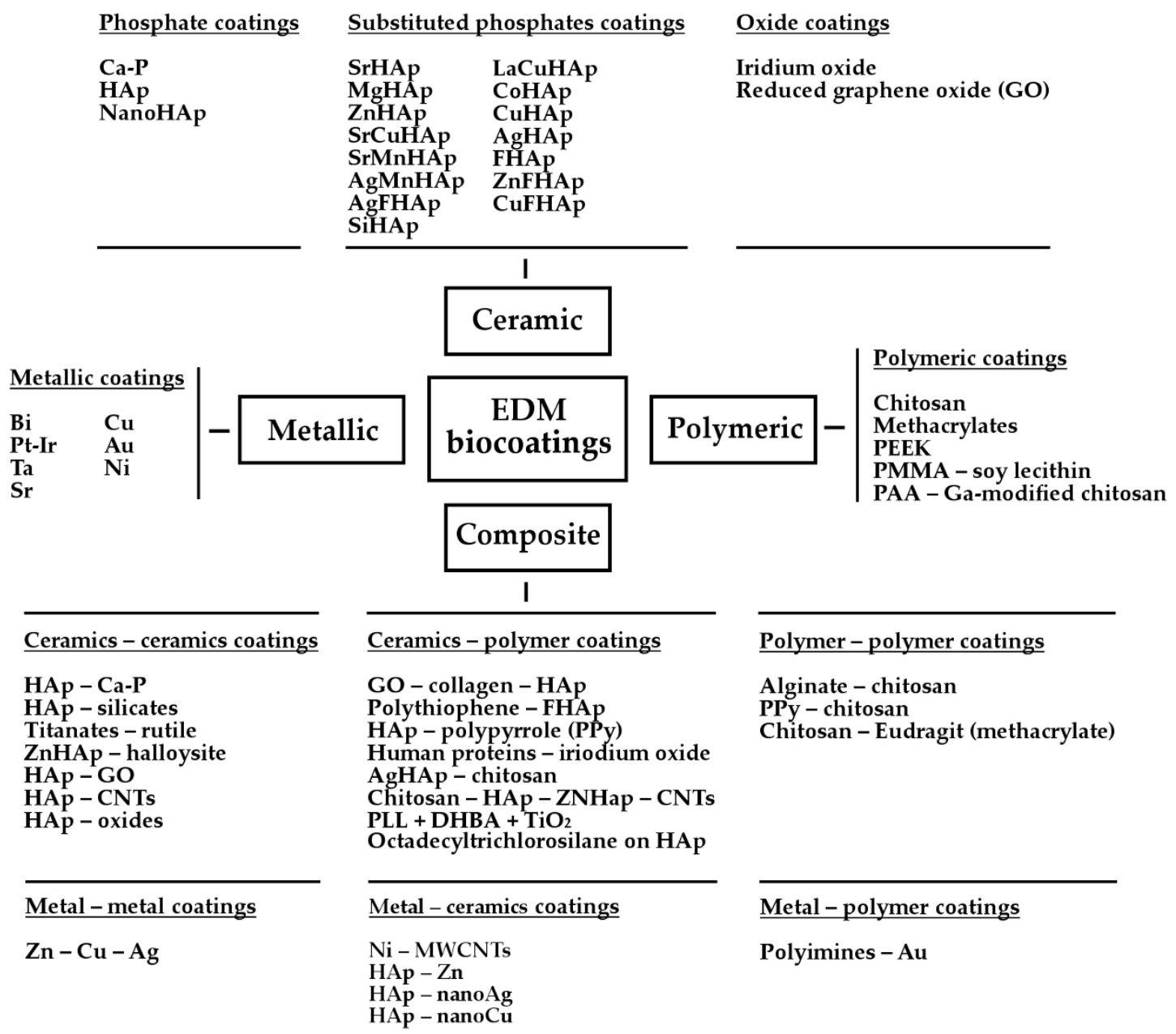

Figure 1. The types of biocoatings created by electrodeposition methods (EDM) as related to the used materials. 


\section{Deposition Technologies}

\subsection{Electrocathodic Deposition (ECD)}

Among the electrodeposition methods, the electrochemical deposition [16], also called (ECAD) or cathodic deposition, seems the most popular. The crucial determinants of microstructure and properties of coatings include an electrolyte composition, voltage or current, depending on electrodeposition mode, and process time. Some research designs and results are described below. The specific determinants for many investigations are shown in Table 1 for one-component metallic, ceramic and polymer, and composite coatings.

Table 1. The main determinants of the electrocathodic deposition of biocoatings.

\begin{tabular}{|c|c|c|c|c|}
\hline Coating; Substrate & Solution and $\mathrm{pH}$ & $\begin{array}{l}\text { Current Density and/or } \\
\text { Voltage Control }\end{array}$ & $\begin{array}{l}\text { Deposition Time } \\
\text { and Temperature }\end{array}$ & Reference \\
\hline \multicolumn{5}{|c|}{ Metallic Coatings } \\
\hline Bismuth & Bismuth nitrate in acetate $\mathrm{pH} 4.5$ & $-1.0 \mathrm{~V}$ & $100 \mathrm{~s}$ & [23] \\
\hline $\begin{array}{l}\mathrm{Cu}-\mathrm{Cu}_{2} \mathrm{O} \text {; graphitic } \\
\text { carbon nitride }\end{array}$ & $\mathrm{H}_{2} \mathrm{SO}_{4}, \mathrm{Cu}_{2} \mathrm{SO}_{4}, \mathrm{Ce}$ and $\mathrm{Ni}$ salts & $-0.8 \mathrm{~V}$ & - & [148] \\
\hline $\mathrm{Ni} ; \mathrm{Ti}$ & nickel sulfate, nickel chloride, boric acid & $50 \mathrm{~mA} / \mathrm{cm}^{2}$ & $\begin{array}{c}\mathrm{RT} \\
15-60 \mathrm{~s}\end{array}$ & [28] \\
\hline $\mathrm{Ni} / \mathrm{Au} ; \mathrm{PC}$ & $\begin{array}{c}\mathrm{NiSO}_{4}, \mathrm{H}_{3} \mathrm{BO}_{3}, \mathrm{KCl} \text { (for } \mathrm{Ni} \text { ) } \\
\mathrm{Au}(\mathrm{CN})_{2} \text { for } \mathrm{Ag}\end{array}$ & $1.4 \mathrm{~V}$ & $1 \mathrm{~min}($ for $\mathrm{Ni}$ ) & [27] \\
\hline Sr; Ti and TiZr & $\begin{array}{l}\text { strontium-acetate and acetic acid, } \mathrm{Sr}-\mathrm{NaF}, \mathrm{Sr}-\mathrm{NaCl} \\
\text { pH } 5\end{array}$ & $1.3 \mathrm{~mA} / \mathrm{cm}^{2}$ & $\begin{array}{l}21^{\circ} \mathrm{C} \\
60 \mathrm{~s}\end{array}$ & [26] \\
\hline Pt-Ir & - & - & - & {$[24]$} \\
\hline \multicolumn{5}{|c|}{ Ceramic Coatings } \\
\hline $\begin{array}{l}\text { Calcium phosphate } \\
\text { (Ca-P); Ti }\end{array}$ & $\begin{array}{c}\mathrm{CaCl}_{2}, \mathrm{Ca}\left(\mathrm{H}_{2} \mathrm{PO}_{4}\right)_{2}, \mathrm{H}_{2} \mathrm{O}_{2} . \\
\text { pH 2.5-6.0 }\end{array}$ & $-0.8 \mathrm{~V}$ vs. SCE. & $\begin{array}{l}1.67-60 \mathrm{~min} \\
45-65^{\circ} \mathrm{C}\end{array}$ & [149] \\
\hline Ca-P; Ti & $\mathrm{NH}_{4} \mathrm{H}_{2} \mathrm{PO}_{4}, \mathrm{Ca}\left(\mathrm{NO}_{3}\right)_{2}$ & - & $37^{\circ} \mathrm{C}$ & [150] \\
\hline $\mathrm{Ca}-\mathrm{P} ; \mathrm{Ti}$ & $\begin{array}{c}\mathrm{NH}_{4} \mathrm{H}_{2} \mathrm{PO}_{4}, \mathrm{Ca}\left(\mathrm{NO}_{3}\right)_{2}, \mathrm{NaNO}_{3} . \\
\mathrm{pH}=7.4\end{array}$ & $3.33 \mathrm{~mA} / \mathrm{cm}^{2}$ & $\begin{array}{l}159 \min \\
24{ }^{\circ} \mathrm{C}\end{array}$ & [106] \\
\hline Ca-P; Ti & $\begin{array}{c}\text { Modified SBF: } \mathrm{NaCl}, \mathrm{NaHCO}_{3}, \\
\mathrm{KH}_{2} \mathrm{PO}_{4} \\
\text { pH } 7.4\end{array}$ & $\begin{array}{c}\text { from }-1.5 \mathrm{~V} \text { to }-2.5 \mathrm{~V} \text { vs. } \\
\text { SCE }\end{array}$ & $\begin{array}{c}1 \mathrm{~h} \\
60^{\circ} \mathrm{C}\end{array}$ & {$[30,31]$} \\
\hline $\begin{array}{l}\text { Ca-P; precalcified } \\
(\mathrm{NaOH}) \mathrm{Ti}\end{array}$ & $\begin{array}{c}\mathrm{Ca}\left(\mathrm{NO}_{3}\right)_{2} \\
\mathrm{NH}_{4} \mathrm{H}_{2} \mathrm{PO}_{4} \\
\mathrm{pH}=6\end{array}$ & $2.5 \mathrm{~mA} / \mathrm{cm}^{2}$ & $\begin{array}{l}60 \min \\
\mathrm{RT}\end{array}$ & [32] \\
\hline Ca-P; Ti scaffold & $\mathrm{CaCl}_{2}, \mathrm{NH}_{4} \mathrm{H}_{2} \mathrm{PO}_{4}$ & - & - & [51] \\
\hline Ca-P; Ti-Ni & $\begin{array}{c}\text { Three different conditions; } \\
\text { (i) } \mathrm{Ca}\left(\mathrm{NO}_{3}\right)_{2} \\
\mathrm{NH}_{4} \mathrm{H}_{2} \mathrm{PO}_{4}, \mathrm{H}_{2} \mathrm{O}_{2} \\
\mathrm{pH}=4.3 \\
\text { (ii) and (iii) as above, } \mathrm{pH}=6\end{array}$ & $\begin{array}{l}\text { (i) }-0.6 \mathrm{~mA} / \mathrm{cm}^{2} \\
\text { (ii) }-0.5 \mathrm{~mA} / \mathrm{cm}^{2} \\
\text { (iii) }-3 \mathrm{~mA} / \mathrm{cm}^{2} \text { for } 1 \mathrm{~s} \\
\text { and reverse current } 0.1 \\
\mathrm{~mA} / \mathrm{cm}^{2} \text { for } 2 \mathrm{~s}\end{array}$ & $\begin{array}{c}\text { (i) } 70{ }^{\circ} \mathrm{C} \\
\text { (ii) and (iii) } 65^{\circ} \mathrm{C}\end{array}$ & [63] \\
\hline $\mathrm{Ca}-\mathrm{P} ; \mathrm{Mg}$ & $\begin{array}{l}\mathrm{Ca}\left(\mathrm{NO}_{3}\right)_{2} \\
\mathrm{KH}_{2} \mathrm{PO}_{4} \\
\mathrm{pH}=4.6\end{array}$ & $3.5 \mathrm{~V}$ & $\begin{array}{l}90 \mathrm{~min} \\
47^{\circ} \mathrm{C}\end{array}$ & [73] \\
\hline Ca-P; Mg alloy & $\begin{array}{c}\mathrm{NaNO}_{3} \\
\mathrm{NH}_{4} \mathrm{H}_{2} \mathrm{PO}_{4}, \mathrm{Ca}\left(\mathrm{NO}_{3}\right)_{2} \\
\mathrm{H}_{2} \mathrm{O}_{2} \\
\text { pH } 5\end{array}$ & $2.5-20 \mathrm{~V}$ & $\begin{array}{l}40-60 \mathrm{~min} \\
20^{\circ} \mathrm{C}\end{array}$ & [72] \\
\hline $\mathrm{Ca}-\mathrm{P} ; \mathrm{Mg}-1 \mathrm{Ca}$ & $\begin{array}{c}\mathrm{Ca}\left(\mathrm{NO}_{3}\right)_{2} \\
\mathrm{NH}_{4} \mathrm{H}_{2} \mathrm{PO}_{4} \\
\mathrm{pH}=5\end{array}$ & -2.5 V vs. SCE & 20-240 min & [67] \\
\hline HAp; Ti & $\begin{array}{c}\mathrm{NH}_{4} \mathrm{H}_{2} \mathrm{PO}_{4}, \mathrm{Ca}\left(\mathrm{NO}_{3}\right)_{2} \\
\mathrm{pH}=7.2\end{array}$ & $-2.5 \mathrm{~V}$ & $\begin{array}{l}10 \mathrm{~min} \\
80^{\circ} \mathrm{C}\end{array}$ & [52] \\
\hline HAp; Ti & $\begin{array}{l}\mathrm{Ca}\left(\mathrm{NO}_{3}\right)_{2} \\
\mathrm{NH}_{4} \mathrm{H}_{2} \mathrm{PO}_{4}\end{array}$ & $-1.8 \mathrm{~V}$ vs. $\mathrm{Ag} / \mathrm{AgCl}$ & $\begin{array}{c}5 \mathrm{~s} \\
80^{\circ} \mathrm{C}\end{array}$ & [33] \\
\hline HAp; Ti & $\begin{array}{l}\text { (i) calcium acetate, } \\
\text { acetic acid. } \\
\text { (ii) } \mathrm{Na}_{3} \mathrm{PO}_{4}, \mathrm{NaOH} \\
\text { pH } 9.1\end{array}$ & $2-4 \mathrm{~V}$ & $1 \mathrm{~h}$ & [151] \\
\hline HAp & $\begin{array}{l}\text { Supersaturated solution of } \mathrm{Ca}\left(\mathrm{NO}_{3}\right)_{2} \text { and } \\
\qquad \mathrm{NH}_{4} \mathrm{H}_{2} \mathrm{PO}_{4} .\end{array}$ & $1.5 \mathrm{~V}$ & $\begin{array}{c}80^{\circ} \mathrm{C} \\
1 \text { and } 4 \mathrm{~h}\end{array}$ & {$[50]$} \\
\hline
\end{tabular}


Table 1. Cont.

\begin{tabular}{|c|c|c|c|c|}
\hline Coating; Substrate & Solution and $\mathrm{pH}$ & $\begin{array}{l}\text { Current Density and/or } \\
\text { Voltage Control }\end{array}$ & $\begin{array}{l}\text { Deposition Time } \\
\text { and Temperature }\end{array}$ & Reference \\
\hline \multicolumn{5}{|c|}{ Ceramic Coatings } \\
\hline HAp; Ti-6Al-4V & $\begin{array}{c}\mathrm{Ca}\left(\mathrm{NO}_{3}\right)_{2}, \mathrm{NH}_{4}\left(\mathrm{H}_{2} \mathrm{PO}_{4}\right) \\
\mathrm{NaNO}_{3} . \\
\mathrm{pH} 4.2\end{array}$ & $0.6 \mathrm{~mA} / \mathrm{cm}^{2}$ & $\begin{array}{l}45 \min \\
\text { RT }\end{array}$ & [75] \\
\hline $\begin{array}{l}\text { HAp; Ti, Ti6Al4V, } \\
\text { stainless steels }\end{array}$ & $\begin{array}{c}\mathrm{Ca}\left(\mathrm{NO}_{3}\right)_{2}, \mathrm{NH}_{4}\left(\mathrm{H}_{2} \mathrm{PO}_{4}\right) \\
\mathrm{NaNO}_{3}, \mathrm{H}_{2} \mathrm{O}_{2} \\
\text { pH } 5.5\end{array}$ & $3 \mathrm{~V}$ & $\begin{array}{c}1 \mathrm{~h} \\
85^{\circ} \mathrm{C}\end{array}$ & [56] \\
\hline HAp; CoCrMo & $\begin{array}{c}\mathrm{Ca}\left(\mathrm{NO}_{3}\right)_{2} \\
\mathrm{NH}_{4} \mathrm{H}_{2} \mathrm{PO}_{4}, \mathrm{H}_{2} \mathrm{O}_{2} \\
\mathrm{pH}=4.5\end{array}$ & $3 \mathrm{~mA} / \mathrm{cm}^{2}$ & $\begin{array}{l}30 \mathrm{~min} \\
20^{\circ} \mathrm{C}\end{array}$ & [61] \\
\hline HAp; CoNiCrMo & $\begin{array}{c}\mathrm{Ca}\left(\mathrm{NO}_{3}\right)_{2} \\
\mathrm{NH}_{4} \mathrm{H}_{2} \mathrm{PO}_{4}\end{array}$ & $\begin{array}{l}\text { From }-1.4 \text { to }-2.2 \mathrm{~V} \\
\text { versus } \mathrm{Ag} / \mathrm{AgCl})\end{array}$ & $80^{\circ} \mathrm{C}$ & {$[60]$} \\
\hline HAp; Mg & $\begin{array}{c}\mathrm{Ca}\left(\mathrm{NO}_{3}\right)_{2} \\
\mathrm{NH}_{4} \mathrm{H}_{2} \mathrm{PO}_{4}, \mathrm{H}_{2} \mathrm{O}_{2} \\
\mathrm{pH}=4.3\end{array}$ & $4 \mathrm{~V}$ & $\begin{array}{l}2 \mathrm{~h} \\
\mathrm{RT}\end{array}$ & [66] \\
\hline $\begin{array}{c}\text { HAp; Au, PC, stainless } \\
\text { steels }\end{array}$ & $\begin{array}{c}\mathrm{Ca}\left(\mathrm{NO}_{3}\right)_{2}, \mathrm{NH}_{4}\left(\mathrm{H}_{2} \mathrm{PO}_{4}\right) \\
\mathrm{H}_{2} \mathrm{O}_{2} \\
\text { pH } 4.5 \text { or } 6 \\
\end{array}$ & $\begin{array}{c}120-250 \mathrm{~mA} / \mathrm{cm}^{2} \\
\text { or }-1.6 \mathrm{~V} \text { vs. } \mathrm{Ag} / \mathrm{AgCl}\end{array}$ & $\begin{array}{l}3-10 \mathrm{~min} \\
70^{\circ} \mathrm{C}\end{array}$ & [152] \\
\hline HAp; enamel & $\mathrm{Ca}\left(\mathrm{NO}_{3}\right)_{2}, \mathrm{NH}_{4} \mathrm{H}_{2} \mathrm{PO}_{4}, \mathrm{NaNO}_{3}$ & $0.5 \mathrm{~mA} / \mathrm{cm}^{2}$ & $\begin{array}{c}1 \mathrm{~h} \\
55^{\circ} \mathrm{C}\end{array}$ & [74] \\
\hline HAp; PVA/PLA & $\mathrm{H}_{2} \mathrm{O}_{2}, \mathrm{CaCl}_{2}, \mathrm{KH}_{2} \mathrm{PO}_{4}$ & $2.5-7.5 \mathrm{~mA} / \mathrm{cm}^{2}$ & $1 \mathrm{~h}$ & {$[78]$} \\
\hline FHAp; stainless steels & $\begin{array}{c}\mathrm{CaCl}_{2} \\
\mathrm{NH}_{4}\left(\mathrm{H}_{2} \mathrm{PO}_{4}\right) \\
\mathrm{NH}_{4} \mathrm{~F}, \mathrm{H}_{2} \mathrm{O}_{2} \\
\mathrm{pH}=4.6\end{array}$ & $1 \mathrm{~mA} / \mathrm{cm}^{2}$ & $\begin{array}{c}1 \mathrm{~min} \\
20-65^{\circ} \mathrm{C}\end{array}$ & [5] \\
\hline $\begin{array}{l}\text { Brushite (DCPD) or } \\
\text { FHAp; } \mathrm{Mg}\end{array}$ & $\begin{array}{c}\mathrm{Ca}\left(\mathrm{NO}_{3}\right)_{2} \\
\mathrm{NH}_{4} \mathrm{H}_{2} \mathrm{PO}_{4} \\
\text { For FHAp, } \mathrm{NaNO}_{3} \text { and } \mathrm{NaF} \text { added. } \\
\mathrm{pH}=4.4\end{array}$ & $\begin{array}{c}5 \text { (DCPD) or } 0.5 \text { (FHAp) } \\
\mathrm{mA} / \mathrm{cm}^{2}\end{array}$ & $\begin{array}{c}\text { RT (DCPD) } \\
\text { or } 60^{\circ} \mathrm{C} \text { (FHAp) }\end{array}$ & [68] \\
\hline AgHAp; Ti & $\begin{array}{c}\mathrm{NaCl} \\
\text { tris(hydroxymethyl)aminomethane, } \mathrm{CaCl}_{2} \text {, } \\
\mathrm{KH}_{2} \mathrm{PO}_{4} \\
\mathrm{pH}=7.2\end{array}$ & $12.5 \mathrm{~mA} / \mathrm{cm}^{2}$ & $95^{\circ} \mathrm{C}$ & [134] \\
\hline AgMnHAp; Ti & $\begin{array}{c}\mathrm{Ca}\left(\mathrm{NO}_{3}\right)_{2} \\
\mathrm{NH}_{4} \mathrm{H}_{2} \mathrm{PO}_{4} \\
\mathrm{Mn}\left(\mathrm{NO}_{3}\right)_{2} \\
\mathrm{AgNO}_{3}\end{array}$ & $0.09 \mathrm{~mA} / \mathrm{cm}^{2}$ & $65^{\circ} \mathrm{C}$ & [85] \\
\hline$(\mathrm{La} / \mathrm{Cu}) \mathrm{HAp}$ & $\begin{array}{c}\mathrm{Ca}\left(\mathrm{NO}_{3}\right)_{2} \\
\mathrm{La}\left(\mathrm{NO}_{3}\right)_{2} \\
\mathrm{Cu}\left(\mathrm{CH}_{3} \mathrm{COO}\right)_{2} \\
\mathrm{NH}_{4} \mathrm{H}_{2} \mathrm{PO}_{4}, \mathrm{H}_{2} \mathrm{O}_{2} \\
\mathrm{pH}=4.5\end{array}$ & $1.0 \mathrm{~mA} / \mathrm{cm}^{2}$ & $\begin{array}{l}1 \mathrm{~h} \\
65^{\circ} \mathrm{C}\end{array}$ & [86] \\
\hline $\begin{array}{c}\text { MgHAp; nanotubular } \\
\mathrm{TiO}_{2}\end{array}$ & $\begin{array}{l}\mathrm{Ca}\left(\mathrm{NO}_{3}\right)_{2} \\
\mathrm{NH}_{4} \mathrm{H}_{2} \mathrm{PO}_{4} \\
\mathrm{Mg}\left(\mathrm{NO}_{3}\right)_{2} \\
\mathrm{pH}=4.2\end{array}$ & $0.85 \mathrm{~mA} / \mathrm{cm}^{2}$ & $\begin{array}{l}35 \mathrm{~min} \\
65^{\circ} \mathrm{C}\end{array}$ & [81] \\
\hline SrHAp; Ti & $\begin{array}{c}\mathrm{CaCl}_{2} \\
\mathrm{NH}_{4}\left(\mathrm{H}_{2} \mathrm{PO}_{4}\right) \\
\mathrm{SrCl}_{2}, \mathrm{NaNO}_{3}\end{array}$ & $3.0 \mathrm{~V}$ & $\begin{array}{c}1 \mathrm{~h} \\
85^{\circ} \mathrm{C}\end{array}$ & [84] \\
\hline SrCuHAp & $\begin{array}{c}\left.\mathrm{Ca}\left(\mathrm{NO}_{3}\right)_{2}, \mathrm{Sr}\left(\mathrm{NO}_{3}\right)_{2}, \mathrm{CuNO}_{3}\right)_{2} \\
\mathrm{NH}_{4} \mathrm{H}_{2} \mathrm{PO}_{4} \\
\mathrm{pH}=4.4\end{array}$ & $0.85 \mathrm{~mA} / \mathrm{cm}^{2}$ & $\begin{array}{l}30 \min \\
65^{\circ} \mathrm{C}\end{array}$ & [82] \\
\hline SrMnHAp & $\begin{array}{c}\mathrm{Ca}\left(\mathrm{NO}_{3}\right)_{2}, \mathrm{Sr}\left(\mathrm{NO}_{3}\right)_{2}, \mathrm{Mn}\left(\mathrm{NO}_{3}\right)_{2} \\
\mathrm{NH}_{4} \mathrm{H}_{2} \mathrm{PO}_{4} \\
\mathrm{pH}=4.3\end{array}$ & $0.85 \mathrm{~mA} / \mathrm{cm}^{2}$ & $\begin{array}{l}30 \min \\
65^{\circ} \mathrm{C}\end{array}$ & [83] \\
\hline $\mathrm{HAp}+\mathrm{CNTs}$ & $\begin{array}{c}\mathrm{Ca}\left(\mathrm{NO}_{3}\right)_{2}, \mathrm{NH}_{4} \mathrm{H}_{2} \mathrm{PO} 4, \mathrm{H}_{2} \mathrm{O}_{2} \\
\text { MWCNTs }\end{array}$ & $3 \mathrm{~V}$ & $\mathrm{pH} 4.7$ & [114] \\
\hline ZnHAp; Ti & $\begin{array}{c}\mathrm{Ca}\left(\mathrm{NO}_{3}\right)_{2} \\
\mathrm{NH}_{4} \mathrm{H}_{2} \mathrm{PO} \\
\mathrm{NaNO}_{3}, \mathrm{H}_{2} \mathrm{O}_{2}\end{array}$ & $2.5 \mathrm{~V}$ & $\begin{array}{l}2 \mathrm{~h} \\
85^{\circ} \mathrm{C}\end{array}$ & [133] \\
\hline ZnHAp; stainless steel & $\begin{array}{c}\mathrm{Ca}\left(\mathrm{NO}_{3}\right)_{2} \\
\mathrm{NH}_{4} \mathrm{H}_{2} \mathrm{PO}_{4}, \mathrm{H}_{2} \mathrm{O}_{2} \\
\mathrm{pH}=4.5\end{array}$ & $0.5-3 \mathrm{~mA} / \mathrm{cm}^{2}$ & $\begin{array}{l}1 \mathrm{~h} \\
65^{\circ} \mathrm{C}\end{array}$ & [54] \\
\hline $\begin{array}{l}\mathrm{CaCO}_{3} ; \text { indium tin } \\
\text { oxide }\end{array}$ & $\begin{array}{c}\mathrm{CaCl}_{2 \prime} \\
\mathrm{NaHCO}_{3}, \mathrm{NaCl} \cdot \mathrm{pH}=8.25\end{array}$ & $-0.86 \mathrm{~V}$ & $25^{\circ} \mathrm{C}$ & [29] \\
\hline
\end{tabular}


Table 1. Cont.

\begin{tabular}{|c|c|c|c|c|}
\hline Coating; Substrate & Solution and $\mathrm{pH}$ & $\begin{array}{l}\text { Current Density and/or } \\
\text { Voltage Control }\end{array}$ & $\begin{array}{l}\text { Deposition Time } \\
\text { and Temperature }\end{array}$ & Reference \\
\hline \multicolumn{5}{|c|}{ Polymer Coatings } \\
\hline Chitosan; Ti-6Al-4V & $\begin{array}{c}\mathrm{CH}_{3} \mathrm{COOH} \\
\text { Chitosan, } \mathrm{NaOH} \\
\mathrm{pH}=4.75\end{array}$ & $0.6 \mathrm{~mA} / \mathrm{cm}^{2}$ & $\begin{array}{l}10 \min \\
\mathrm{RT}\end{array}$ & [96] \\
\hline $\begin{array}{l}\text { Poly } \\
\text { (DL-lactide-co-glycolide) } \\
\text { (PLGA); stainless steel }\end{array}$ & PLGA solution & $2 \mathrm{~mA}$ & - & [97] \\
\hline \multicolumn{5}{|c|}{ Composite Coatings } \\
\hline Ni-MWCNTs & $\begin{array}{c}\mathrm{NiSO}_{4}, \mathrm{NiCl}_{2} \\
\mathrm{H}_{3} \mathrm{BO}_{3} \text {, saccharine }\end{array}$ & $80 \mathrm{~mA} / \mathrm{cm}^{2}$ & - & [132] \\
\hline $\mathrm{Pd} / \mathrm{Ag} / \mathrm{HAp}$ & $\mathrm{NH}_{4} \mathrm{H}_{2} \mathrm{PO} 4, \mathrm{NH}_{4} \mathrm{~F}, \mathrm{HAp}, \mathrm{Pd}, \mathrm{Ag}$ & $23 \mathrm{~V}$ & $1 \mathrm{~h}$ & [139] \\
\hline NanoHAp-CNTs & $\begin{array}{c}\text { CNTs, } \mathrm{NH}_{4} \mathrm{H}_{2} \mathrm{PO}_{4}, \mathrm{Ca}\left(\mathrm{NO}_{3}\right)_{2}, \mathrm{NaNO}_{3} . \\
\mathrm{pH}=7.4\end{array}$ & $5 \mathrm{~mA}$ & $\begin{array}{c}15-30 \mathrm{~min} \\
100{ }^{\circ} \mathrm{C}\end{array}$ & [77] \\
\hline CNTs-HAp & $\begin{array}{c}\mathrm{Ca}\left(\mathrm{NO}_{3}\right)_{2} \\
\mathrm{~K}_{2} \mathrm{HPO}_{4}, \mathrm{CNTs}\end{array}$ & $-1.4 \mathrm{~V}$ vs. SCE & $1 \mathrm{~h}$ & [112] \\
\hline $\mathrm{HAp}-\mathrm{CaSiO}_{3}$ & $\begin{array}{c}\text { nano- } \mathrm{SiO}_{2} \\
\mathrm{Ca}\left(\mathrm{NO}_{3}\right)_{2} \\
\mathrm{NH}_{4} \mathrm{H}_{2} \mathrm{PO}_{4} \\
\mathrm{pH}=4.2\end{array}$ & $0.8 \mathrm{~mA} / \mathrm{cm}^{2}$ & $\begin{array}{l}30 \min \\
65^{\circ} \mathrm{C}\end{array}$ & [104] \\
\hline $\begin{array}{l}\text { HAp-CaHPO } \\
\text { stainless steels }\end{array}$ & $\begin{array}{c}\mathrm{CaCl}_{2} \\
\mathrm{NH}_{4} \mathrm{H}_{2} \mathrm{PO}_{4} \\
\end{array}$ & $\begin{array}{c}5 \text { or } 10 \mathrm{~mA} / \mathrm{cm}^{2} \\
1 \mathrm{~V}, 2 \mathrm{~V} \text { or3 } \mathrm{V}\end{array}$ & RT & [58] \\
\hline $\mathrm{ZnHAp}-\mathrm{CaSiO}_{3}$ & $\begin{array}{c}\mathrm{Ca}\left(\mathrm{NO}_{3}\right)_{2} \\
\mathrm{NH}_{4} \mathrm{H}_{2} \mathrm{PO}_{4}, \mathrm{Zn}\left(\mathrm{NO}_{3}\right)_{2} \\
\mathrm{pH}=4.2\end{array}$ & $0.8 \mathrm{~mA} / \mathrm{cm}^{2}$ & $\begin{array}{l}30 \min \\
65^{\circ} \mathrm{C}\end{array}$ & [105] \\
\hline $\begin{array}{l}\mathrm{ZnO} / \mathrm{ZnHAp} \text { hybrid } \\
\text { coating; } \\
\text { carbon fiber }\end{array}$ & $\begin{array}{r}\mathrm{Zn}\left(\mathrm{NO}_{3}\right)_{2} \\
\mathrm{Ca}\left(\mathrm{NO}_{3}\right)_{2} \\
\mathrm{NH}_{4} \mathrm{H}_{2} \mathrm{PO}_{4}\end{array}$ & $\begin{array}{l}\text { 1st stage: } 0.6 \mathrm{~mA} / \mathrm{cm}^{2} \\
\text { (ECD). } \\
\text { 2nd stage: } 3 \mathrm{~V}(\mathrm{EPD})\end{array}$ & $\begin{array}{c}\text { 1st stage: } 30 \mathrm{~min} \text {, } \\
343 \mathrm{~K} \\
\text { 2nd stage: } 60 \mathrm{~min}\end{array}$ & [119] \\
\hline $\begin{array}{c}\text { Zn-halloysite } \\
\text { nanotubes } \\
\text { (HNT)/SrSmHAp } \\
\text { hybride coating; } \\
\text { Ti6Al4V }\end{array}$ & $\begin{array}{c}\mathrm{Ca}\left(\mathrm{NO}_{3}\right)_{2}, \\
\mathrm{Sr}\left(\mathrm{NO}_{3}\right)_{2}, \mathrm{Sm}\left(\mathrm{NO}_{3}\right)_{2}, \mathrm{NH}_{4} \mathrm{H}_{2} \mathrm{PO}_{4}\end{array}$ & $1.0 \mathrm{~mA} / \mathrm{cm}^{2}$ & $\begin{array}{l}30 \min \\
\text { RT }\end{array}$ & [108] \\
\hline $\begin{array}{l}\text { Halloysite nanotubes } \\
\text { (HNT)-CeHAp; Ti } \\
\text { alloy }\end{array}$ & $\begin{array}{c}\mathrm{Ca}\left(\mathrm{NO}_{3}\right)_{2}, \mathrm{NH}_{4} \mathrm{H}_{2} \mathrm{PO}_{4} \\
\mathrm{Ce}\left(\mathrm{NO}_{3}\right)_{2}, \text { halloysite nanoclay, } \mathrm{HCl} . \\
\text { pH }=4.5\end{array}$ & - & - & [107] \\
\hline $\begin{array}{l}\text { HAp-Ag-chitosan; Pt, } \\
\text { graphite or stainless } \\
\text { steel }\end{array}$ & $\begin{array}{l}\text { chitosan } \\
\text { solutions containing } \mathrm{HAp} \text { nanoparticles and } \\
\text { dissolved } \mathrm{AgNO}_{3}\end{array}$ & $0.1 \mathrm{~mA} / \mathrm{cm}^{2}$ & - & [125] \\
\hline $\mathrm{HAp}-\mathrm{ZrO}{ }_{2} ; \mathrm{Ti}$ & $\begin{array}{c}\mathrm{Ca}\left(\mathrm{NO}_{3}\right)_{2}, \mathrm{NH}_{4} \mathrm{H}_{2} \mathrm{PO}_{4}, \mathrm{NaNO}_{3}, \mathrm{H}_{2} \mathrm{O}_{2}, \mathrm{ZrO}_{2} \\
\text { particles. pH }=4.5\end{array}$ & $1 \mathrm{~mA} / \mathrm{cm}^{2}$ & $\begin{array}{l}45 \min \\
65^{\circ} \mathrm{C}\end{array}$ & [102] \\
\hline $\mathrm{HAp}-\mathrm{ZrO}_{2}-\mathrm{TiO}_{2}$ & $\begin{array}{c}\mathrm{Ca}\left(\mathrm{NO}_{3}\right)_{2} \\
\mathrm{NH}_{4} \mathrm{H}_{2} \mathrm{PO}_{4} \\
\mathrm{NaNO}_{3}, \mathrm{ZrO}_{2}, \mathrm{TiO}_{2} \\
\mathrm{pH}=4.2\end{array}$ & Constant direct current & $\begin{array}{l}85^{\circ} \mathrm{C} \\
2 \mathrm{~h}\end{array}$ & [118] \\
\hline $\begin{array}{l}\text { HAp-GO-collagen; } \\
\text { Ti-Nb }\end{array}$ & $\begin{array}{c}\mathrm{Ca}\left(\mathrm{NO}_{3}\right)_{2}, \mathrm{NH}_{4} \mathrm{H}_{2} \mathrm{PO}_{4}, \mathrm{GO} \text {, collagen in SBF. } \\
\mathrm{pH}=4.1-4.3\end{array}$ & $2 \mathrm{~mA} / \mathrm{cm}^{2}$ & $\begin{array}{l}60 \min \\
33^{\circ} \mathrm{C}\end{array}$ & [122] \\
\hline $\begin{array}{l}\text { GO (an inner } \\
\text { layer)/GO-MgHAp (an } \\
\text { outer layer); C/C } \\
\text { composites }\end{array}$ & $\begin{array}{l}\text { 1st stage (GO inner-layer): Go water suspension. } \\
\text { 2nd stage (GO-MGHAp): } \mathrm{NH}_{4} \mathrm{H}_{2} \mathrm{PO}_{4}, \mathrm{Ca}\left(\mathrm{NO}_{3}\right)_{2} \\
\qquad \mathrm{Mg}\left(\mathrm{NO}_{3}\right)_{2}, \mathrm{GO}\end{array}$ & $\begin{array}{l}\text { 1st stage: } 30-70 \mathrm{~V} \text { (EPD). } \\
\text { 2nd stage: } 3 \mathrm{~mA} \text { (ECD). }\end{array}$ & $\begin{array}{l}\text { 1st stage: } 1-7 \mathrm{~min} \\
\text { (EPD). } \\
\text { 2nd stage: } 1 \mathrm{~h}, 50 \\
{ }^{\circ} \mathrm{C} \text { (ECD). }\end{array}$ & [111] \\
\hline $\begin{array}{l}\text { Chitosan-AgHAp on } \\
\text { nanotubular } \mathrm{TiO}_{2}\end{array}$ & $\begin{array}{c}\mathrm{Ca}\left(\mathrm{NO}_{3}\right)_{2} \\
\mathrm{NH}_{4} \mathrm{H}_{2} \mathrm{PO}_{4} \\
\mathrm{AgNO}_{3}\end{array}$ & $\begin{array}{c}0.85 \mathrm{~mA} / \mathrm{cm}^{2} \\
35 \mathrm{~min} \text { at } 50 \circ \mathrm{C}\end{array}$ & $\begin{array}{l}35 \mathrm{~min} \\
50^{\circ} \mathrm{C}\end{array}$ & [126] \\
\hline $\begin{array}{l}\text { Poliethyleneimine } \\
\text { (PEI)-Ag }\end{array}$ & Hydrogen tetrachlorate (III). & -1.2 V vs. (Ag/AgCl) & $45 \mathrm{~s}$ & [142] \\
\hline $\begin{array}{l}\text { Polypyrrole-chitosan; } \\
\text { stainless steel }\end{array}$ & $\begin{array}{l}\text { Pyrrole in oxalic acid, with and without the } \\
\text { addition of chitosan. }\end{array}$ & $15 \mathrm{~mA}$ & $1 \mathrm{~h}$ & [144] \\
\hline $\begin{array}{l}\text { Polyacrylic acid (PAA) } \\
\text { followed by } \\
\text { Ga-modified chitosan; } \\
\text { Ti }\end{array}$ & PAA water solution & $\begin{array}{l}\text { For Ga-modified } \\
\text { chitosan: } 1.5 \mathrm{~V}\end{array}$ & $15-60 \mathrm{~min}$ & [146] \\
\hline $\begin{array}{c}\text { Alginate/chitosan } \\
\text { (layer-by-layer coating) }\end{array}$ & $\begin{array}{l}\text { Chitosan water solution } \\
\text { Alginate dissolved in acetic acid }\end{array}$ & $20 \mathrm{~V}$ & $20 \mathrm{~min}$ & [143] \\
\hline Chitosan-protein; Mg & $\begin{array}{l}\text { Chitosan in acetic acid } \\
\text { Proteins in citric acid }\end{array}$ & $1 \mathrm{~mA} / \mathrm{cm}^{2}$ & $10 \mathrm{~min}$ & [98] \\
\hline
\end{tabular}

RT-room temperature. Empty spaces mean that no data have been reported in references. 


\subsubsection{Effect of an Electrolyte Composition}

As shown in Table 1, the regular baths for the deposition of Ca-P compounds contained such salts as $\mathrm{Ca}\left(\mathrm{NO}_{3}\right)_{2}, \mathrm{CaCl}_{2}, \mathrm{CaSiO}_{3}$, and $\left(\mathrm{NH}_{4}\right) \mathrm{H}_{2} \mathrm{PO}_{4}$, often $\mathrm{H}_{2} \mathrm{O}_{2}$, more seldom $\mathrm{KH}_{2} \mathrm{PO}_{4}$. The typical concentration of calcium salt was $0.042 \mathrm{~mol} / \mathrm{L}$ and $0.025 \mathrm{~mol} / \mathrm{L}$ of $\left(\mathrm{NH}_{4}\right) \mathrm{H}_{2} \mathrm{PO}_{4}$. Hydrogen peroxide was added in concentration from $2000 \mathrm{ppm}$ to even $2 \%$ [61]. As concerns the other additives, in [114] the addition of $1 \%$ MWCNTs to HAp showed the most positive effect on corrosion resistance.

\subsubsection{Effect of Deposition Potential}

In deposition performed under constant voltage, its value was usually between 1 and $3 \mathrm{~V}$. The impact of potential was investigated in a few works. In [58], at $1 \mathrm{~V}$ of voltage, 300 to $400 \mathrm{~nm}$ thick flake type of deposition with random orientation was observed together with flakes either parallel or perpendicular to the coating surfaces, and under 2 and $3 \mathrm{~V}$ potential, an average thickness of flakes increased to around $2 \mu \mathrm{m}$. In [33,60], calcium phosphate coatings on the CoNiCrMo substrate were prepared by electrodeposition at different voltages and the moderate potential sample $(-1.8 \mathrm{~V}$ vs. $\mathrm{AgCl})$ they exhibited the most uniform coating. In [30], the calcium phosphates were electrodeposited on titanium and the coating electrodeposited at $-2.0 \mathrm{~V}$ (SCE; Saturated Calomel Electrode) in the modified SBF containing $\mathrm{CO}_{3}{ }^{2-}$ ions was the most bioactive, showing transformation into carbonate apatite similar to bone apatite. As stated in [152], the desired potential range for HAp electrodeposition may be comprised between -1.5 and $-2.2 \mathrm{~V}(\mathrm{Ag} / \mathrm{AgCl})$. These results are like each other and the effect of potential seems moderate.

\subsubsection{Effect of Deposition Current}

Such studies were carried out usually at current densities beginning well below $1 \mathrm{~mA} / \mathrm{cm}^{2}$ up to even $10 \mathrm{~mA} / \mathrm{cm}^{2}$ or more, as shown in Table 1. In [58], under constant cathode current density of $5 \mathrm{~mA} / \mathrm{cm}^{2}$, the broom-like shape of deposition structure was observed, with flakes precisely perpendicular to the coating surfaces, with one after another originated from a single point with a wall thickness of around $400-500 \mathrm{~nm}$, and spherical-shaped porous apatite structure with a diameter around $800-900 \mathrm{~nm}$ along with $50-100 \mathrm{~nm}$ pores throughout the outer peripheral surface appeared. At $10 \mathrm{~mA} / \mathrm{cm}^{2}$ current density, the continuous coating, and random flake type deposits were observed with around 1-2 $\mu \mathrm{m}$ wall thickness. In the ultrasonic-assisted electrodeposition of hydroxyapatite [78], when the current density increased from 2.5 to $7.5 \mathrm{~mA} / \mathrm{cm}^{2}$, the grain size decreased from 414 to $265 \mathrm{~nm}$. With the current density increasing from 2.5 to $5.0 \mathrm{~mA} / \mathrm{cm}^{2}$, the $\mathrm{Ca} / \mathrm{P}$ ratio rises from 1.53 to 1.58. Conversely, the $\mathrm{Ca} / \mathrm{P}$ ratio drastically decreases to 1.25 at a current density of $7.5 \mathrm{~mA} / \mathrm{cm}^{2}$.

\subsubsection{Effect of Deposition Time}

The result of deposition time was not often investigated, and applied values were diverse. In [34], by increasing the electrodeposition time from 1 to $30 \mathrm{~min}$, the coating thickness increased, but also the surface morphology of the Ca-P coatings was greatly affected going from smooth to plate-like, featuring elongated plates, ribbon-like to finally sharp needle structures. The optimum electrodeposition procedures leading to both good cell-material interaction and sufficient mechanical properties could be achieved with relatively thin coatings produced at short electrodeposition times. The loading time increased the HAp crystallinity [149].

\subsubsection{Effect of Deposition $\mathrm{pH}$}

The $\mathrm{pH}$ value, a rule, was buffered between 4 and 6 . The HAp crystallization process was favored by an increase in $\mathrm{pH}$ from 2.5 to 6 [149]. 


\subsubsection{Effect of Deposition Temperature}

The deposition processes were conducted either at room temperature or, seldom, about $60-70^{\circ} \mathrm{C}$. In [149], with increasing temperature, the deposited hydroxyapatite was occasionally of plate-like shape, and the width and the length of the deposited calcium phosphates at $65^{\circ} \mathrm{C}$ were more extensive than those at $55^{\circ} \mathrm{C}$.

\subsection{Pulse Electrocathodic Deposition (PED)}

The important determinants are shown in Table 2 for one-component metallic, ceramic and polymer, and composite coatings.

Table 2. The main determinants of the pulse electrocathodic deposition of biocoatings.

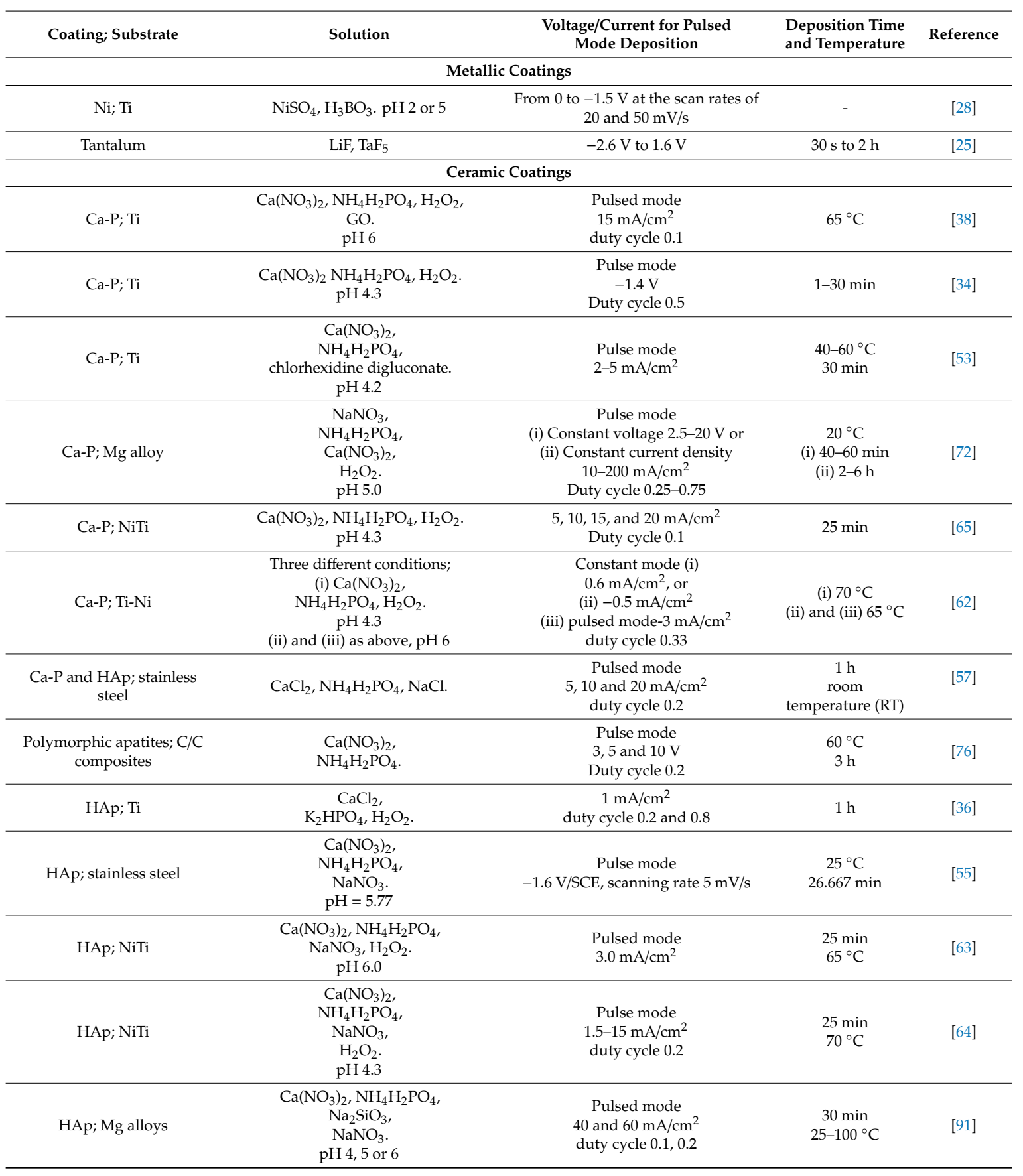


Table 2. Cont.

\begin{tabular}{|c|c|c|c|c|}
\hline Coating; Substrate & Solution & $\begin{array}{l}\text { Voltage/Current for Pulsed } \\
\text { Mode Deposition }\end{array}$ & $\begin{array}{l}\text { Deposition Time } \\
\text { and Temperature }\end{array}$ & Reference \\
\hline \multicolumn{5}{|c|}{ Ceramic Coatings } \\
\hline NanoHAp; Mg alloy & $\begin{array}{c}\mathrm{Ca}\left(\mathrm{NO}_{3}\right)_{2}, \mathrm{NH}_{4} \mathrm{H}_{2} \mathrm{PO}_{4} \\
\mathrm{H}_{2} \mathrm{O}_{2} \\
\mathrm{pH}=4.5\end{array}$ & $\begin{array}{l}\text { Pulse mode } \\
\quad-3 \mathrm{~V} \\
\text { Duty cycle } 0.2\end{array}$ & RT & [69] \\
\hline NanoHAp; Mg-Zn scaffold & $\begin{array}{c}\mathrm{Ca}\left(\mathrm{NO}_{3}\right)_{2}, \mathrm{NH}_{4} \mathrm{H}_{2} \mathrm{PO}_{4} \\
\mathrm{NaNO}_{3}\end{array}$ & $\begin{array}{l}20-40 \mathrm{~mA} / \mathrm{cm}^{2} \\
\text { Duty cycle } 0.1 \text { and } 0.2 \\
\text { temperature, }\end{array}$ & $\begin{array}{c}55,70,85 \text { and } \\
100{ }^{\circ} \mathrm{C} \\
1 \mathrm{~h} \\
\end{array}$ & [70] \\
\hline NanoHAp; Mg-Zn scaffolds & $\begin{array}{c}\mathrm{Ca}\left(\mathrm{NO}_{3}\right)_{2} \\
\mathrm{NH}_{4} \mathrm{H}_{2} \mathrm{PO}_{4} \\
\mathrm{NaNO}_{3} \\
\mathrm{pH}=5.0\end{array}$ & $\begin{array}{c}40 \mathrm{~mA} / \mathrm{cm}^{2} \\
\text { duty cycle } 0.1\end{array}$ & $\begin{array}{l}85^{\circ} \mathrm{C} \\
1 \mathrm{~h}\end{array}$ & [71] \\
\hline HAp; Au & $\begin{array}{c}\mathrm{Ca}\left(\mathrm{NO}_{3}\right)_{2} . \mathrm{NH}_{4} \mathrm{H}_{2} \mathrm{PO}_{4} \\
\mathrm{H}_{2} \mathrm{O}_{2} \\
\text { pH } 4.5 \text { or } 6\end{array}$ & $\begin{array}{c}\text { Constant mode: } 1.6 \mathrm{~V} \text { vs. } \mathrm{Ag} / \mathrm{AgCl} \\
\text { followed by a pulsed mode } \\
\text { duty cycle } 0.33\end{array}$ & $\begin{array}{l}45 \mathrm{~min} \\
70^{\circ} \mathrm{C}\end{array}$ & [152] \\
\hline $\mathrm{HAp}$; nanoTiO 2 & $\mathrm{Ca}\left(\mathrm{NO}_{3}\right)_{2}, \mathrm{NH}_{4} \mathrm{H}_{2} \mathrm{PO}_{4}$ & $\begin{array}{c}2.5 \mathrm{~mA} / \mathrm{cm}^{2} \\
\text { Duty cycle } 0.5\end{array}$ & $20-120 \mathrm{~s}$ & [49] \\
\hline $\begin{array}{l}\text { CoCa-P; } \\
\text { Ti22Nb6Zr }\end{array}$ & $\begin{array}{l}\mathrm{Ca}\left(\mathrm{NO}_{3}\right)_{2}, \mathrm{NH}_{4} \mathrm{H}_{2} \mathrm{PO}_{4} \\
\quad \mathrm{Co}\left(\mathrm{NO}_{3}\right)_{2}, \mathrm{H}_{2} \mathrm{O}_{2}\end{array}$ & $\begin{array}{l}\text { Pulsed mode } \\
15 \mathrm{~mA} / \mathrm{cm}^{2}\end{array}$ & $15 \min$ & [87] \\
\hline FHAp; $\mathrm{Mg}-\mathrm{Zn}-\mathrm{Ca}$ & $\begin{array}{c}\mathrm{NaNO}_{3} \\
\mathrm{NH}_{4} \mathrm{H}_{2} \mathrm{PO}_{4} \\
\mathrm{Ca}\left(\mathrm{NO}_{3}\right)_{2}, \mathrm{NaF}_{2} \mathrm{H}_{2} \mathrm{O}_{2} . \\
\text { pH } 5.0\end{array}$ & $\begin{array}{l}\text { Pulse mode } \\
1 \mathrm{~mA} / \mathrm{cm}^{2}\end{array}$ & $65^{\circ} \mathrm{C}$ & [89] \\
\hline SiHAp; Mg alloy & $\begin{array}{c}\mathrm{Ca}\left(\mathrm{NO}_{3}\right)_{2}, \mathrm{NH}_{4} \mathrm{H}_{2} \mathrm{PO}_{4}, \\
\mathrm{NaNO}_{3} \\
\text { tetraethoxysilane. }\end{array}$ & $\begin{array}{c}\text { Pulse mode } \\
0.4-0.6 \mathrm{~V} \\
\text { Duty cycle } 0.3\end{array}$ & $\begin{array}{l}40-80^{\circ} \mathrm{C} \\
40 \mathrm{~min}\end{array}$ & [90] \\
\hline (Zn,Mg,Sr,Ag)Ca-P; Ti6Al4V & $\begin{array}{c}\mathrm{Ca}\left(\mathrm{NO}_{3}\right)_{2} \\
\mathrm{NH}_{4} \mathrm{H}_{2} \mathrm{PO}_{4}, \mathrm{AgNO}_{3} \\
\mathrm{Zn}\left(\mathrm{NO}_{3}\right)_{2}, \mathrm{Sr}\left(\mathrm{NO}_{3}\right)_{2}, \mathrm{Mg}\left(\mathrm{NO}_{3}\right)_{2} \\
\mathrm{H}_{2} \mathrm{O}_{2}\end{array}$ & $\begin{array}{l}\text { Pulsed mode } \\
400 \mathrm{~mA} / \mathrm{cm}^{2} \\
\text { duty cycle } 0.2\end{array}$ & $70^{\circ} \mathrm{C}$ & [88] \\
\hline ZnHAp & $\begin{array}{c}\mathrm{Ca}\left(\mathrm{NO}_{3}\right)_{2}, \mathrm{NH}_{4} \mathrm{H}_{2} \mathrm{PO}_{4}, \mathrm{H}_{2} \mathrm{O}_{2} . \\
\text { pH } 4.5\end{array}$ & $\begin{array}{l}\text { Pulsed mode } \\
0.5-3 \mathrm{~mA} / \mathrm{cm}^{2}\end{array}$ & $\begin{array}{l}1 \mathrm{~h} \\
65^{\circ} \mathrm{C} \\
\end{array}$ & [95] \\
\hline $\mathrm{HAp}+\mathrm{CNTs}$ & $\begin{array}{c}\mathrm{Ca}\left(\mathrm{NO}_{3}\right)_{2}, \mathrm{NH}_{4} \mathrm{H}_{2} \mathrm{PO} 4, \mathrm{H}_{2} \mathrm{O}_{2} \\
\text { MWCNTs }\end{array}$ & $3 \mathrm{~V}$ & $\mathrm{pH} 4.7$ & [95] \\
\hline \multicolumn{5}{|c|}{ Composite Coatings } \\
\hline $\begin{array}{c}\text { Reduced graphene oxide } \\
\text { (rGO)-polydopamine- } \\
\text { CuNPs-Nil blue; glass } \\
\text { carbon }\end{array}$ & $\begin{array}{c}\mathrm{Cu}\left(\mathrm{NO}_{3}\right)_{2} \\
\text { phosphate-buffered saline (PBS). }\end{array}$ & $\begin{array}{c}\text { Pulse mode } \\
\text { from }-0.5 \mathrm{~V} \text { to } 0.8 \mathrm{~V}, \\
\text { a scan rate of } 100 \mathrm{mV} / \mathrm{s}\end{array}$ & - & [95] \\
\hline $\begin{array}{l}\text { MCWNT-HAp; stainless } \\
\text { steel }\end{array}$ & $\begin{array}{l}\mathrm{CaCl}_{2}, \mathrm{NH}_{4} \mathrm{H}_{2} \mathrm{PO}_{4} \\
\mathrm{NaCl}\end{array}$ & $\begin{array}{c}\text { Pulsed mode } \\
5,10, \text { and } 20 \mathrm{~mA} / \mathrm{cm}^{2} \\
\text { duty cycle } 0.2\end{array}$ & $\begin{array}{l}1 \mathrm{~h} \\
\mathrm{RT}\end{array}$ & [103] \\
\hline $\begin{array}{c}\mathrm{HAp}-\mathrm{CaHPO} \\
\text { steel }\end{array}$ & $\begin{array}{c}\mathrm{CaCl}_{2} \\
\mathrm{NH}_{4} \mathrm{H}_{2} \mathrm{PO}_{4}\end{array}$ & $\begin{array}{c}\text { Pulsed mode } \\
\text { Either constant current } \\
5 \text { and } 10 \mathrm{~mA} / \mathrm{cm}^{2} \text {, or constant voltage } \\
1,2 \text { and } 3 \mathrm{~V}\end{array}$ & RT & [58] \\
\hline
\end{tabular}


Table 2. Cont.

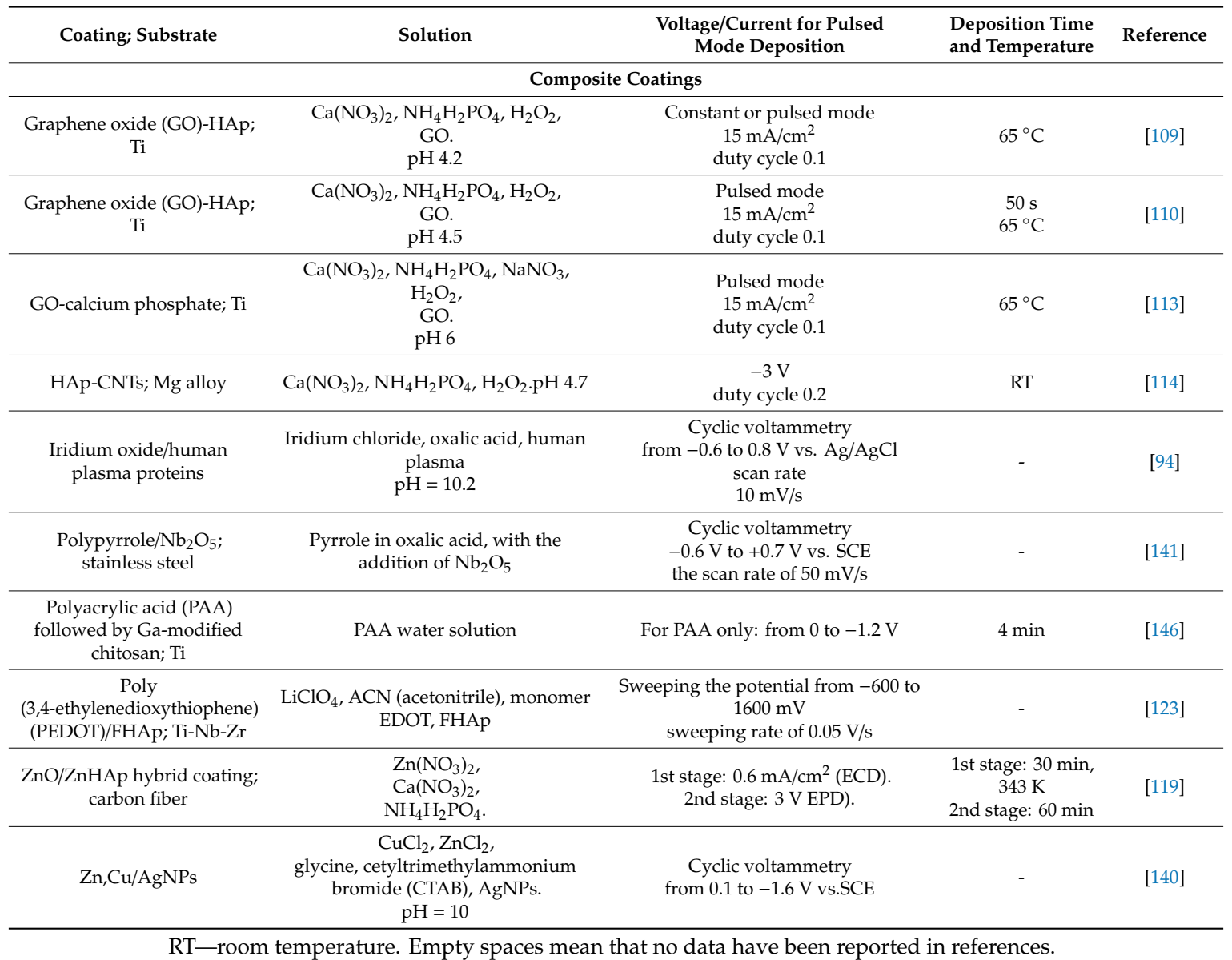

\subsubsection{Effect of an Electrolyte Composition}

As for the ECD, applied electrolytes were prepared based on $0.042 \mathrm{~mol} / \mathrm{L}$ of $\mathrm{Ca}\left(\mathrm{NO}_{3}\right)_{2}$ and $0.025 \mathrm{~mol} / \mathrm{L}$ of $\mathrm{NH}_{4}\left(\mathrm{H}_{2} \mathrm{PO}_{4}\right)$. Exceptionally, the SBF solution [58] was used during deposition $\left(\mathrm{NaCl}-7.996 \mathrm{~g} / \mathrm{L}, \mathrm{KCl} \longrightarrow 0.224 \mathrm{~g} / \mathrm{L}, \mathrm{CaCl}_{2} \cdot 2 \mathrm{H}_{2} \mathrm{O}-0.278 \mathrm{~g} / \mathrm{L}, \mathrm{MgCl}_{2} \cdot 6 \mathrm{H}_{2} \mathrm{O} \longrightarrow 0.305 \mathrm{~g} / \mathrm{L}, \mathrm{NaHCO}_{3} \longrightarrow 0.350 \mathrm{~g} / \mathrm{L}\right.$, $\mathrm{K}_{2} \mathrm{HPO}_{4} \cdot 3 \mathrm{H}_{2} \mathrm{O}-0.228 \mathrm{~g} / \mathrm{L}$, and $\mathrm{Na}_{2} \mathrm{SO}_{4}-0.071 \mathrm{~g} / \mathrm{L}$ ). The increasing $\mathrm{Ca}$ and $\mathrm{P}$ contents in an electrolyte affected the morphology of HAp [49], from separated particle to plate-like.

Hydroxyapatite, carbonate apatite, and dicalcium phosphate dehydrate were deposited on carbon/carbon (C/C) composites [76]. The supersaturation degrees of $\mathrm{Ca}^{2+}$ and $\mathrm{PO}_{4}{ }^{3-}$ changed the crystalline habit of HAp, led to large crystal size, and transformed crystal shape from belt-like to a plant-like structure. The $\mathrm{H}_{2} \mathrm{O}_{2}$ was added to prevent hydrogen bubbles formation during electrodeposition and to favor better calcium phosphate crystals nucleation [152]. For HAp coatings deposited on NiTi alloy by PED, the morphology of the coating changed from needle-like to plate-like structure as the electrolyte concentration decreased about five times [64].

\subsubsection{Effect of Deposition Potential}

The deposition under constant voltage was usually run by cyclic voltammetry between to fixed potential value, come and back. In some reports [152], the pulsed mode was applied, in which the voltage was imposed for $60 \mathrm{~s}$ followed by break-time for $120 \mathrm{~s}$. During the deposition of hydroxyapatite, carbonate apatite, and dicalcium phosphate dehydrate on C/C composites [76], the increasing voltage resulted in promoting bioactivity by altering the morphologies and phases. For silver nanoparticles in solution during the electrodeposition of $\mathrm{Zn}-\mathrm{Cu} / \mathrm{AgNP}$ composite coatings by cyclic voltammetry [140], the process was shown to occur through two stages with different energies. The first stage occurred in 
the potential range from -0.4 to $-0.7 \mathrm{~V}$ vs. SCE and was mainly associated with the electrodeposition of a copper film, while the second stage corresponded to the bulk deposition of $\mathrm{Zn}-\mathrm{Cu} / \mathrm{AgNPs}$ and occurred from -1.4 to $-1.6 \mathrm{~V}$ vs. SCE. The increasing HAp nucleation rate vis-à-vis increasing deposition potential was observed, and the presence of calcium orthophosphate phase was decreased along [58] with an increased rate of hydroxyapatite phase in the coating.

\subsubsection{Effect of Deposition Current}

Using the current pulsed methods, smooth uniform coatings were obtained onto Mg alloy when a current density in a specific range was used $\left(10-30 \mathrm{~mA} / \mathrm{cm}^{2}\right)$ [72]. The growth of particles was observed when low frequencies $(50 \mathrm{~Hz})$ and high frequencies $(1000 \mathrm{~Hz})$ were applied.

In [58], the effect of current parameters was observed. In other research [91], when Si-HAp coating was deposited on Mg-5Zn-0.3Ca alloy substrate by pulse electrodeposition, at a low current density of $20 \mathrm{~mA} / \mathrm{cm}^{2}$ a coarse and non-uniform coating was deposited, and at a high current density of $60 \mathrm{~mA} / \mathrm{cm}^{2}$, high amounts of hydrogen gas at the interface were produced, and a non-uniform coating was formed. At the middle current density of $40 \mathrm{~mA} / \mathrm{cm}^{2}$, nano-needle like coating was observed. For higher peak current density, the Ca-P coating consisting of needle-shaped crystals was formed with pores in between [54]. At lower current density of $0.5 \mathrm{~mA} / \mathrm{cm}^{2}$ a more compact uniform plate-like morphology was observed than those at a higher current density of $3 \mathrm{~mA} / \mathrm{cm}^{2}$.

In [152], by the template-assisted pulsed electrodeposition method, the calcium-deficient hydroxyapatite (CDHA) particles in aqueous baths with hydrogen peroxide were used by both applying pulsed current density and pulsed potential in cathodic electrodeposition. Deposition in the membrane was successful only for higher values of current density, so a valid value of $120-250 \mathrm{~mA} / \mathrm{cm}^{2}$ was applied. In other research [101], a composite coating of hydroxyapatite and calcium hydrogen phosphate over 316 grade of stainless steel performed by PED at $10 \mathrm{~mA} / \mathrm{cm}^{2}$ demonstrated the highest weight percentage and crystallinity of hydroxyapatite phase and a continuous, faster and interconnected cell growth. For a composite coating of hydroxyapatite-polypyrrole, the coating deposited with moderate current density $\left(10 \mathrm{~mA} / \mathrm{cm}^{2}\right)$ seems to be the optimum one regarding the faster-interconnected growth of MG63 cells over the coating surface along with highest corrosion resistance and anodic passivation capability [124]. In [65], the electrodeposition at the higher current densities of 15 and $20 \mathrm{~mA} / \mathrm{cm}^{2}$ increased the possibility of the hydroxyapatite phase formation in the coating rather than the other less stable calcium phosphate phases. The optimum conditions to create a uniform nano-hydroxyapatite coating on the Mg-Zn scaffold was found at $40 \mathrm{~mA} / \mathrm{cm}^{2}$ and 0.1 duty cycle [71]. Finally, in research [63], in which reverse current densities of $0.1 \mathrm{~mA} / \mathrm{cm}^{2}$ were applied, the porosity was increased by increasing the current density of the reverse pulse. Also, in this condition, the resulted film was composed of nanosized HA crystals. It seems that the reverse current step can influence the porosity and the structure of the coatings by the dissolution of the unstable phases, which are formed during the direct current stage of the bipolar pulse deposition. For the production of acceptable quality Ca-P coatings on $\mathrm{Mg}$ alloy, the current density should be between 10 and $30 \mathrm{~mA} / \mathrm{cm}^{2}$ [72]. In [49], the observed HAp morphologies consisted of particles, a mixture of particles and plate-like shapes, and entirely plate-like shapes as related to the increasing number of deposition cycles.

\subsubsection{Effect of Deposition $\mathrm{pH}$}

The $\mathrm{pH}$ value was generally acidic. For deposited SiHAp coating at $\mathrm{pH} 5$, the needle-like morphology, but the coatings deposited at $\mathrm{pH} 4$ and 6, the plate-like morphology with micro-size thickness were observed [91].

\subsubsection{Effect of Deposition Temperature}

The applied were either room temperature or elevated temperatures, like $40-60^{\circ} \mathrm{C}$ [53], $85^{\circ} \mathrm{C}$ [70], or even $200{ }^{\circ} \mathrm{C}$ [25] temperatures were reported. In [91], tests were made at temperatures ranging 
from 25 to $100{ }^{\circ} \mathrm{C}$, at temperatures up to $85^{\circ} \mathrm{C}$, the morphology of the SiHAp coating changed to the nano-sized needle-like blades.

\subsection{Electrophoretic Deposition (EPD)}

Table 3 illustrates the process determinants of several investigations performed by EPD.

Table 3. The main determinants of the electrophoretic deposition of biocoatings.

\begin{tabular}{|c|c|c|c|c|}
\hline Coating; Substrate & Electrolyte & Voltage/Current & $\begin{array}{l}\text { Deposition Time } \\
\text { and Temperature }\end{array}$ & Reference \\
\hline HAp; NiTi & ethanol & $40 \mathrm{~V}$ & $20 \mathrm{~s}$ & [39] \\
\hline $\mathrm{HAp} ; \mathrm{TiO}_{2}$ & - & $200 \mathrm{~V}$ & $1 \mathrm{~min}$ & {$[44]$} \\
\hline НAp; Ti & ethanol & $10-45 \mathrm{~V}$ & $1-8 \mathrm{~min}$ & [42] \\
\hline $\mathrm{HAp} ; \mathrm{TiO}_{2}$ & ethanol & $60 \mathrm{~V}$ & $\begin{array}{l}45 \mathrm{~s} \\
\mathrm{RT}\end{array}$ & [45] \\
\hline $\mathrm{HAp} ; \mathrm{Au}$ & $\begin{array}{l}\text { Ethanol and octadecyltri- } \\
\text { chlorosilane }\end{array}$ & $70 \mathrm{~V} / \mathrm{cm}^{2}$ & $1 \mathrm{~h}$ & [131] \\
\hline $\mathrm{Hap}+\mathrm{TiO}_{2}$ & acetylacetone & $20 \mathrm{~V}$ & $30-120 \mathrm{~s}$ & {$[43]$} \\
\hline HAp + CNTs; NiTi & ethanol & $30 \mathrm{~V}$ & $30 \mathrm{~s}$ & [116] \\
\hline Hap + MWCNTs & butanol & $60 \mathrm{~V}$ & $2 \mathrm{~min}$ & {$[117]$} \\
\hline Hap + MNWCNTs + nanoAg + nanoCu & ethanol, isopropanol & 11 and $30 \mathrm{~V}$ & $\begin{array}{l}2 \min \\
\mathrm{RT}\end{array}$ & [138] \\
\hline nanoHAp; Ag & ethanol & $10 \mathrm{~V}$ & & [47] \\
\hline Nano(Zn/Ca)HAp; $(\mathrm{Si}) \mathrm{Ti}$ & - & $\begin{array}{l}\text { 10, 50, and } 100 \\
\mathrm{~V} / \mathrm{cm}\end{array}$ & $\begin{array}{l}1 \mathrm{~min} \\
\mathrm{pH} 12\end{array}$ & [93] \\
\hline nanoHAp; Ti & ethanol & $10 \mathrm{~V}$ & $10 \mathrm{~min}$ & [41] \\
\hline nanoHAp; $(\mathrm{Mg}, \mathrm{Zr}, \mathrm{Ce})$ oxides & water & $380 \mathrm{~V}$ & $10 \mathrm{~min}$ & [46] \\
\hline nanoHAp & $0.1,0.2$ or $0.5 \mathrm{~g}$ nanoHAp & 15,30 , and $50 \mathrm{~V}$ & $1 \mathrm{~min}$ & {$[40]$} \\
\hline nanoHAp + nanoAg & ethanol & $\begin{array}{l}15 \text { and } 30 \mathrm{~V} \text { for } \\
\text { nanoHAp } \\
60 \mathrm{~V} \text { for nanoAg }\end{array}$ & $\begin{array}{c}1 \text { min for } \\
\text { nanoHAp } \\
5 \text { min for nanoAg }\end{array}$ & [135] \\
\hline nanoHAp + nanoAg & ethanol & $50 \mathrm{~V}$ & $1 \mathrm{~min}$ & [136] \\
\hline nanoHAp + nanoCu; TiO2 & ethanol & $30 \mathrm{~V}$ & $\begin{array}{l}1 \text { and } 2 \text { min } \\
\text { RT }\end{array}$ & [137] \\
\hline nanoHAp + borium nitride; Ti & ethanol & 100 and $150 \mathrm{~V}$ & $\begin{array}{l}5-20 \mathrm{~s} \\
\mathrm{pH} 4\end{array}$ & [120] \\
\hline PEEK + HAp & ethanol & $75 \mathrm{~V}$ & $\begin{array}{c}45 \mathrm{~s} \\
\mathrm{pH} 5.5\end{array}$ & [130] \\
\hline $\mathrm{HAp}+\mathrm{Si}+\mathrm{MWCNTs}$ & - & cathodic & - & {$[153]$} \\
\hline Chitosan + HAp; $\mathrm{TiO}_{2}$ & acetic acid, etanol, water & $10-15 \mathrm{~V}$ & $3-9 \min$ & {$[154]$} \\
\hline Hap + ZNHAp + MWCNTs + chitosan & - & - & - & {$[128]$} \\
\hline Bioglass + HAp (whiskers) & isopropanol & $40 \mathrm{~V}$ & $1 \mathrm{~min}$ & [121] \\
\hline Chitosan + bioglass + HAp & acetic acid, etanol, water & 20 and $30 \mathrm{~V}$ & $\begin{array}{l}5 \text { and } 15 \text { min } \\
\mathrm{pH} 3.3,4,5\end{array}$ & [155] \\
\hline $\begin{array}{c}\mathrm{GO} \text { (graphene oxide) + MgHAp; C/C } \\
\text { composites }\end{array}$ & - & - & - & [111] \\
\hline $\begin{array}{c}\text { The poly-l-lysine (PLL) + } \\
\text { 3,4-dihydroxybenzylaldehyde (DHBA) + } \\
\mathrm{HAp}+\mathrm{TiO}_{2}\end{array}$ & ethanol-water & $50 \mathrm{~V}$ & - & [129] \\
\hline methacrylates & dioxan & $15 \mathrm{~V}$ & $5 \mathrm{~min}$ & [99] \\
\hline PEEK & ethanol & $70-115 \mathrm{~V}$ & $1 \mathrm{~min}$ & {$[100]$} \\
\hline Chitosan + Eudragit & acetic acid & 10 and $30 \mathrm{~V}$ & 1 and $3 \mathrm{~min}$ & [145] \\
\hline PMMA + soy lecithin & $\begin{array}{l}\text { the microemulsion of } \\
\text { coconut oil and water }\end{array}$ & $1 \mathrm{~mA}(4-15 \mathrm{~V})$ & $\begin{array}{l}30 \mathrm{~min} \\
\mathrm{RT}\end{array}$ & [147] \\
\hline
\end{tabular}

RT-room temperature. Empty spaces mean that no data have been reported in references. 


\subsubsection{Effect of an Electrolyte Composition}

The electrolyte composition affected biological properties. Galindo et al. [93] studied the effect of different $\mathrm{Zn} / \mathrm{Ca}$ ratio on cytocompatibility and antibacterial efficiency demonstrating the best results at the $5 \%$ and $10 \%$ content of $\mathrm{Zn}$ in the carbonated hydroxyapatite (CHAp). In-vitro bioactivity of PEEK-HAp coatings was enhanced by increasing the amount of HAp particles, but such coatings had inferior adhesion to the substrate [130].

The electrolyte also affected corrosion resistance, adhesion, and wettability. The corrosion current, between 1 and $10 \mathrm{nA} / \mathrm{cm}^{2}$, was significantly higher for undecorated nanoHAp coatings and close to that of the substrate for decorated nanoHAp coatings [135]. The compact structure of hydroxyapatite with $20 \mathrm{wt} . \%$ silicon and hydroxyapatite with $20 \mathrm{wt} . \%$ silicon- $1 \mathrm{wt} . \%$ multi-walled carbon nano-tubes coatings could efficiently increase the corrosion resistance of NiTi substrate [153].

The presence of nanosilver particles in the coating improved adhesive properties and reduce contact angle values [136]. Besides, the presence of nanosilver particles has a significant effect on homogeneity and quality of coating. In the presence of the silver nanoparticles, a smaller number of cracks and a smaller dimension of cracks were observed [40]. On the other hand, in [120] the increase in the amount of $\mathrm{BN}$ was insignificant as concerns the coating thickness.

In [121], the electrophoretic deposition of bioactive glass (BG) coatings reinforced by whisker hydroxyapatite (WHA) particles at 50 and $75 \mathrm{wt} . \%$ WHA showed the highest bonding strength and bioactivity response. Addition of other ceramics, MWCNTs, changed mechanical properties [117]: the hardness and adhesion strength of the HA coating improved from $72 \mathrm{HV}$ and $17.2 \mathrm{MPa}$ to $405 \mathrm{HV}$ and 32.1 MPa. Moreover, the results of the biological tests revealed an incredible improvement in the apatite and bone cell growth on the HA coatings composed of titanium and MWCNTs. For a duplex coating composed of electrophoretically deposited graphene oxide (GO), inner-layer and electrodeposited GO/Mg substituted hydroxyapatite (MH) outer-layer on carbon/carbon composites (CC) demonstrated the bonding strength between the duplex coating and CC as of $7.4 \mathrm{MPa}$, about $80 \%$ higher than without GO [111]. The composition of HAp and MWCNTs promoted also the osteoblast adhesion and proliferation [129] and bioactivity [116].

The size of HPa seems to play an important role in biological behavior; the cell adhesion was better on the small- and middle-sized nanocrystals (SHA, MHA > LHA), which was interestingly opposite in cell spreading (LHA > SHA, MHA), and then the cell proliferation was again up-regulated on SHA and MHA [42].

\subsubsection{Effect of Deposition Potential}

In [42], the increasing potential at EPD of nanoHAp resulted in increasing coating thickness, the release rate of silver in SBF, resistance to scratch, but in decreasing hardness. On the contrary, in other reports [120], the voltage effect was insignificant as concerns the thickness.

\subsubsection{Effect of Deposition Time}

The increase in deposition time results in an increased thickness, decreasing hardness and increasing adhesion strength, and significantly decreasing the contact angle in wettability tests [137]. When EPD was made on the nanotubular $\mathrm{TiO}_{2}$ surface, at a shorter $1 \mathrm{~min}$ EPD time, hydroxyapatite was deposited inside the nanotubes, between nanotubes, and also formed a thin layer over the nanotubes. At a longer $2 \mathrm{~min}$, EPD time, the layer of $\mathrm{HAp}$ over the $\mathrm{HAp}-\mathrm{TiO}_{2}$ composite was thicker. The increasing thickness with deposition time was observed earlier [120].

\subsection{Plasma Electrochemical Oxidation (PEO)}

The plasma electrochemical oxidation, also called micro-arc oxidation (MAO), is generally assumed as a tool to generate a surface with high roughness affecting the adhesion of osteoblasts. However, $\mathrm{PEO}$, when made in an electrolyte composed of different compounds, may significantly change the 
surface chemical and phase composition. Therefore, the attempts were made to obtain bioactive and well-adjacent to the substrate coatings using this approach.

The significant part of such research was performed on titanium substrates. In [156], the coatings, consisting of HAp and titanium oxide, were produced on Ti6Al4V alloy in a solution containing calcium acetate and $\beta$-calcium glycerophosphate. The phases of anatase, rutile, TCP $\left(\mathrm{Ca}_{3}\left(\mathrm{PO}_{4}\right)_{2}\right)$, perovskite-CaTiO 3 , and $\mathrm{HAp}$ were detected, their crystallinity more profound with increasing treatment time. The friction and wear resistance properties, and corrosion resistance of the PEO coatings were substantially improved compared to Ti6Al4V alloy. In similar research [157], the $\mathrm{HAp} / \mathrm{TiO}_{2}$ composite coating was prepared on a titanium surface. The flocculent structures were obtained during the early stages of treatment and, as the treatment period extended, increasing amounts of Ca-P precipitate appeared on the surface, and the flocculent morphology transformed into a plate-like morphology, and finally into flower-like apatite. The $\mathrm{Ca} / \mathrm{P}$ atomic ratio gradually decreased, and the adhesive strength between the apatite and $\mathrm{TiO}_{2}$ coating was improved. The search for a new complexing agent was described in [158]. The barium titanate coatings were also obtained [159] on Ti6Al4V alloy, and the coatings presented lower friction coefficient, higher wear, and corrosion resistance than uncoated Ti alloys. In [160], the coatings formed in a solution containing Ca and P achieved a thickness of $11.78 \mu \mathrm{m}$, and bond strength $33.69 \mathrm{MPa}$. Recently [161], two-layer hydroxyapatite $\mathrm{CuHAp}-\mathrm{TiO}_{2}$ composite coating was prepared on titanium in an aqueous electrolyte containing $\mathrm{Ca}, \mathrm{P}$, and $\mathrm{Cu}$ at a constant current density of $100 \mathrm{~mA} / \mathrm{cm}^{2}$. Yu et al. [48] obtained Mg-containing hydroxyapatite coatings on Ti-6Al-4V alloy for dental materials by plasma electrolytic oxidation. The increasing Mg content in an electrolyte resulted in decreasing the irregularity of the surface, pore size, and the number of pores decreased as the $\mathrm{Mg}$ concentration increased.

After 20 min of process, $\mathrm{Cu}$ species incorporated into the $\mathrm{TiO}_{2}$ as $\mathrm{CuO}$ and $\mathrm{Cu}_{2} \mathrm{O}$, resulting in the formation of $\mathrm{Cu}$-doped $\mathrm{TiO}_{2}$-based coating. Dziaduszewska et al. [162] found the best process parameters in a similar electrolyte as of $300 \mathrm{~V}$ for $15 \mathrm{~min}$, at 32 or $50 \mathrm{~mA}$ of current value, which resulted in the proper microstructure, high $\mathrm{Ca} / \mathrm{P}$ ratio, hydrophilicity, early-stage bioactivity. The coating mechanical properties, Young's modulus, and hardness were close to the values characteristic for bones, and adhesion was also better than at $200 \mathrm{~V}$ of voltage.

In addition to HAp coatings on Ti, also other substrates were covered with HAp for biological applications. The HAp coatings were grown on Ta samples by PEO at the potential in a range of 350 to $500 \mathrm{~V}$, and treatment time from 60 to $600 \mathrm{~s}$ [163]. The formation of crystalline HAp was detected for samples treated over $180 \mathrm{~s}$ at $500 \mathrm{~V}$. In [164], the coating was obtained by $\mathrm{PEO}$ on $\mathrm{Zr}$ in for process duration of 2 to $30 \mathrm{~min}$. The increasing oxidation resulted in thicker and rougher coatings. The increasing oxidation resulted in thicker and rougher coatings. In [165], a hydrophilic surface was obtained by PEO on $\mathrm{Mg}$ with a biosynthesized hyaluronic acid and carboxymethylcellulose to improve bonding function.

\subsection{Electro-Spark Deposition (ESD)}

The ESD method was carried out on Ti6Al4V to deposit a layer on the steel substrates at the first step, and then the PEO process was employed to improve properties of the alloyed titanium layer at the second step [166]. The duplex coating consisted of $\alpha-\mathrm{Al}_{2} \mathrm{O}_{3}$ (corundum) and $\gamma-\mathrm{Al}_{2} \mathrm{O}_{3}$ phases were formed after the PEO process while $\mathrm{AlFe}_{3}, \mathrm{TiN}$, and $\mathrm{AlTi}_{3}$ phases were detected in the ESD coating. In the next research [167], the HAp coatings were formed on steel again by ESD and PEO. The HAp-based surface was rough and porous, indicated a hydrophilic character, and improved bioactivity in SBF.

\subsection{Electro-Discharge Method (EDM)}

In [168], the hydroxyapatite coating was synthesized on the Zr-based bulk metallic glass surface to promote cell proliferation. The formation of the nanoporous coating of about $27.2 \mu \mathrm{m}$ thick appeared, firmly adhered to adhesive failure at $132 \mathrm{~N}$. A significant rise in cell viability was also shown. In [169], 
the surface modification of the $\beta$-type titanium substrate was made by EDT to incorporate MWCNTs and $\mu$-hydroxyapatite $(\mu \mathrm{HAp})$ powders in the dielectric medium to promote cell proliferation.

\subsection{Electropolymerization (EP)}

The electropolymerization is a method applied for some organic coatings. In [170], MWCNTspolyaniline composite films were prepared by in situ electrochemical polymerizations on the titanium from an aniline solution containing a small content of well-dispersed CNTs. Then these composite films were employed as a substrate for the electrodeposition of platinum nanoparticles forming the surface highly active for electrocatalytic oxidation of glycerol. In a more recent work [171], the polypyrrole (PPy)/chitosan composite coating on titanium was obtained by EP method showing enhanced microhardness and adhesion strength and higher corrosion resistance compared to the PPy coating.

Some specific environments were also utilized. In [172], the enzyme laccase was immobilized during the potentiostatic deposition of a thin polydopamine film (PDA) on carbon surfaces.

\subsection{Effects of Electrodeposition Method on Properties of Coatings}

The electrodeposition methods are important, even not prevalent (the sprayed coatings are the mostly applied for joint implants) for coatings on orthopedic and dental implants, maxillofacial implants, and stents. However, the choice of method depends on the designed coating, its predicted properties, and applications.

The most popular are electrocathodic deposition at either constant or pulse voltage/current, and as the third, electrophoretic deposition. The same coatings, mainly ceramic or composite ceramics-ceramics, ceramics-polymer, or ceramics-metal can be obtained by each of these methods. Therefore, the research was conducted on similar coatings by these methods even if there were few attempts to compare the coatings of the identical composition obtained by all EDM techniques.

ECD is the simplest and most developed technique in the past. However, it is excellent only for some metals. As concerns ceramics which cannot dissociate to ions, their deposition on the metal base is difficult, the surfaces are often non-stoichiometric and non-homogenous. Despite that, it is still the most popular deposition method, thanks to its simplicity, and it is widely used for Ca-P and HAp coatings, one or multicomponent layers. The applied voltage is between -1.5 to $2.5 \mathrm{~V}$ (SCE) for the Ti based materials and a little more for Mg alloys. The process is usually made under voltage control, and for current control, the current values are $0.1-3 \mathrm{~mA} / \mathrm{cm}^{2}$. Deposition time depends on a used compound, and it is very short for metals, 15-60 s, and much longer for ceramics, For Ca-P and HAp coatings, even up to $1 \mathrm{~h}$. The used bath is based mainly on two substances, $\mathrm{Ca}\left(\mathrm{NO}_{3}\right)_{2}$ and $\mathrm{NH}_{4} \mathrm{H}_{2} \mathrm{PO}_{4}$. The process parameters seem to play a less important role: the electrolyte composition is determined by the ionic components of designed coating, the voltage must be maintained within some limits, $\mathrm{pH}$ is usually neutral or slightly acidic. The ECD gives then no great possibility to adjust the properties of coatings by process parameters.

The pulse electrocathodic deposition (PED) gives more possibilities to control the coating. The voltage or, more seldom, current, is the obvious process parameter, but a duty cycle and number of cycles (deposition time) allow creating of previously design coating. The electric parameters are like those used under direct current, the duty cycle is between 0.1 and 0.5 , deposition time reaches even $1 \mathrm{~h}$.

The electrophoretic deposition is exceptionally plausible for no dissociated compounds such as ceramics and polymers. For the PED, process parameters such as duty cycle and the number of cycles need some preliminary tests. For the EPD, the most significant difficulty is to use the proper bath components (together with their contents in a bath) and solvent, in which a stable suspension is obtained and the species possess the proper charge. Ethanol, butanol, and isopropanol are mainly applied, but this list can likely be extended in the future affecting the process efficiency and coating adhesion and thickness. It is noteworthy that for the attempts to look for the most optimum electrochemical conditions: voltage was changed between 10 and $100 \mathrm{~V}$, and once even it was $380 \mathrm{~V}$, and deposition 
time was usually short, between $15 \mathrm{~s}$ and $2 \mathrm{~min}$, sometimes longer. The use of such a short time allows obtaining the thin coatings, particularly suitable for coatings on metallic implants, for which an excessive thickness may result in a weak adhesion and failure. Therefore, it is a great advantage of EPD.

The critical factor for adhesion is the surface roughness. As a rule, it is adjusted by mechanical polishing, chemical etching, oxidation of Ti to nanotubular structure, and others. However, the micro-arc oxidation, MAO (called also plasma electrochemical oxidation, PEO) is the unique among electrodeposition methods, as it brings out the rough surface rich in biological elements due to high voltage oxidation in electrolytes containing $\mathrm{Ca}$ and $\mathrm{P}$ compounds. For that reason, it is commonly used for dental implants.

Other methods, such as electro-spark, electrodeposition, and electropolymerization techniques are less used. Despite that, they can be more intensively developed in the future as they allow creating the duplex coatings, nanoporous coatings, and polymer coatings.

\section{Conclusions}

The coatings proposed for medical applications are, to a greater extent, either ceramic or ceramic-ceramic or ceramic-polymer and, to a lesser extent, either polymer, metallic, and other composite coatings such as ceramic-metallic, ceramic-polymer, polymer-polymer, and others.

The most applied compounds are phosphates, and the most used substrates are titanium and its alloys and, to a lesser extent, NiTi alloys.

Three electrocrystallization methods are preferred for deposition of biocoatings such as direct and pulse electrocathodic deposition, and electrophoretic deposition. Plasma electrochemical oxidation, electro-spark, electro-discharge, and electropolymerization are not often applied.

The main process determinants include potential or current, and electrolyte composition, more seldom deposition time, $\mathrm{pH}$, and temperature.

The current progress in biocoatings focuses on an enhancement of their bioactivity, biocompatibility (hydrophilicity), adhesion to substrates, mechanical strength, and corrosion resistance. Such properties are determined by microstructure and thickness of coatings, and characteristics of the interface.

Future research is to be directed towards the development of biocoatings possessing efficient and long term antibacterial efficiency, with no short and long term cytotoxicity, enhancing adhesion, proliferation, and viability of cells, well adjacent to the substrate, while being resistant to shear and compressive stresses, environmentally friendly, and economically justified.

Author Contributions: Conceptualization, A.Z. and M.B.; formal analysis, A.Z.; investigation, A.Z. and M.B.; writing-original draft preparation, A.Z. and M.B. All authors have read and agreed to the published version of the manuscript.

Funding: This research received no external funding.

Conflicts of Interest: The authors declare no conflict of interest.

\section{References}

1. Nakaya, M.; Uedono, A.; Hotta, A. Recent progress in gas barrier thin film coatings on PET bottles in food and beverage applications. Coatings 2015, 5, 987-1001. [CrossRef]

2. Aranke, O.; Algenaid, W.; Awe, S.; Joshi, S. Coatings for automotive gray cast iron brake discs: A review. Coatings 2019, 9, 552. [CrossRef]

3. Ma, L.; Eom, K.; Geringer, J.; Jun, T.S.; Kim, K. Literature review on fretting wear and contact mechanics of tribological coatings. Coatings 2019, 9, 501. [CrossRef]

4. Verbič, A.; Gorjanc, M.; Simončič, B. Zinc oxide for functional textile coatings: Recent advances. Coatings 2019, 9, 550. [CrossRef]

5. Bir, F.; Khireddine, H.; Touati, A.; Sidane, D.; Yala, S.; Oudadesse, H. Electrochemical depositions of fluorohydroxyapatite doped by $\mathrm{Cu}^{2+}, \mathrm{Zn}^{2+}, \mathrm{Ag}^{+}$on stainless steel substrates. Appl. Surf. Sci. 2012, 258, 7021-7030. [CrossRef] 
6. Tiwari, A.; Seman, S.; Singh, G.; Jayaganthan, R. Nanocrystalline cermet coatings for erosion-corrosion protection. Coatings 2019, 9, 400. [CrossRef]

7. Mandracci, P.; Mussano, F.; Rivolo, P.; Carossa, S. Surface treatments and functional coatings for biocompatibility improvement and bacterial adhesion reduction in dental implantology. Coatings 2016, 6, 7 . [CrossRef]

8. Hou, N.Y.; Perinpanayagam, H.; Mozumder, M.S.; Zhu, J. Novel development of biocompatible coatings for bone implants. Coatings 2015, 5, 737-757. [CrossRef]

9. Graziani, G.; Boi, M.; Bianchi, M. A review on ionic substitutions in hydroxyapatite thin films: Towards complete biomimetism. Coatings 2018, 8, 269. [CrossRef]

10. Cometa, S.; Bonifacio, M.A.; Mattioli-Belmonte, M.; Sabbatini, L.; De Giglio, E. Electrochemical strategies for titanium implant polymeric coatings: The why and how. Coatings 2019, 9, 268. [CrossRef]

11. Sartori, M.; Maglio, M.; Tschon, M.; Aldini, N.N.; Visani, A.; Fini, M. Functionalization of ceramic coatings for enhancing integration in osteoporotic bone: A systematic review. Coatings 2019, 9, 312. [CrossRef]

12. Duta, L.; Popescu, A.C. Current status on pulsed laser deposition of coatings from animal-origin calcium phosphate sources. Coatings 2019, 9, 335. [CrossRef]

13. Paital, S.R.; Dahotre, N.B. Calcium phosphate coatings for bio-implant applications: Materials, performance factors, and methodologies. Mater. Sci. Eng. R Rep. 2009, 66, 1-70. [CrossRef]

14. Guslitzer-Okner, R.; Mandler, D. Electrochemical coating of medical implants. In Applications of Electrochemistry and Nanotechnology 291 in Biology and Medicine I; Eliaz, N., Ed.; Springer Science \& Business Media: Berlin, Germany, 2011; pp. 291-342. ISBN 9781461403470.

15. Kulkarni, M.; Mazare, A.; Schmuki, P.; Iglič, A. Biomaterial surface modification of titanium and titanium alloys for medical applications. In Nanomedicine; Seifalian, A., de Mel, A., Kalaskar, D.M., Eds.; One Central Press Altrincham: Cheshire, UK, 2014; pp. 111-136.

16. Asri, R.I.M.; Harun, W.S.W.; Hassan, M.A.; Ghani, S.A.C.; Buyong, Z. A review of hydroxyapatite-based coating techniques: Sol-gel and electrochemical depositions on biocompatible metals. J. Mech. Behav. Biomed. Mater. 2016, 57, 95-108. [CrossRef]

17. Dorozhkin, S.V. Calcium orthophosphates $\left(\mathrm{CaPO}_{4}\right)$ : Occurrence and properties. Prog. Biomater. 2016, 5, 9-70. [CrossRef]

18. Adeleke, S.A.; Bushroa, A.R.; Sopyan, I. Recent development of calcium phosphate-based coatings on titanium alloy implants. Surf. Eng. Appl. Electrochem. 2017, 53, 419-433. [CrossRef]

19. Liu, W.; Liu, S.; Wang, L. Surface modification of biomedical titanium alloy: Micromorphology, microstructure evolution and biomedical applications. Coatings 2019, 9, 249. [CrossRef]

20. Su, Y.; Cockerill, I.; Zheng, Y.; Tang, L.; Qin, Y.X.; Zhu, D. Biofunctionalization of metallic implants by calcium phosphate coatings. Bioact. Mater. 2019, 4, 196-206. [CrossRef]

21. Yang, J.; Cui, F.; Lee, I.S. Surface modifications of magnesium alloys for biomedical applications. Ann. Biomed. Eng. 2011, 39, 1857-1871. [CrossRef]

22. Wan, P.; Tan, L.; Yang, K. Surface modification on biodegradable magnesium alloys as orthopedic implant materials to improve the bio-adaptability: A review. J. Mater. Sci. Technol. 2016, 32, 827-834. [CrossRef]

23. Ananthi, A.; Kumar, S.S.; Phani, K.L. Facile one-step direct electrodeposition of bismuth nanowires on glassy carbon electrode for selective determination of folic acid. Electrochim. Acta 2015, 151, 584-590. [CrossRef]

24. Cassar, I.R.; Yu, C.; Sambangi, J.; Lee, C.D.; Whalen, J.J.; Petrossians, A.; Grill, W.M. Electrodeposited platinum-iridium coating improves in vivo recording performance of chronically implanted microelectrode arrays. Biomaterials 2019, 205, 120-132. [CrossRef] [PubMed]

25. Arnould, C.; Delhalle, J.; Mekhalif, Z. Multifunctional hybrid coating on titanium towards hydroxyapatite growth: Electrodeposition of tantalum and its molecular functionalization with organophosphonic acids films. Electrochim. Acta 2008, 53, 5632-5638. [CrossRef]

26. Frank, M.J.; Walter, M.S.; Tiainen, H.; Rubert, M.; Monjo, M.; Lyngstadaas, S.P.; Haugen, H.J. Coating of metal implant materials with strontium. J. Mater. Sci. Mater. Med. 2013, 24, 2537-2548. [CrossRef]

27. Mollamahale, Y.B.; Ghorbani, M.; Dolati, A.; Hosseini, D. Electrodeposition of well-defined gold nanowires with uniform ends for developing 3D nanoelectrode ensembles with enhanced sensitivity. Mater. Chem. Phys. 2018, 213, 67-75. [CrossRef] 
28. Nasirpouri, F.; Cheshideh, H.; Samardak, A.Y.; Ognev, A.V.; Zubkov, A.A.; Samardak, A.S. Morphologyand magnetism-controlled electrodeposition of $\mathrm{Ni}$ nanostructures on $\mathrm{TiO}_{2}$ nanotubes for hybrid $\mathrm{Ni} / \mathrm{TiO}_{2}$ functional applications. Ceram. Int. 2019, 45, 11258-11269. [CrossRef]

29. Pavez, J.; Silva, J.F.; Melo, F. Homogeneous calcium carbonate coating obtained by electrodeposition: In situ atomic force microscope observations. Electrochim. Acta 2005, 50, 3488-3494. [CrossRef]

30. Park, J.H.; Lee, D.Y.; Oh, K.T.; Lee, Y.K.; Kim, K.M.; Kim, K.N. Bioactivity of calcium phosphate coatings prepared by electrodeposition in a modified simulated body fluid. Mater. Lett. 2006, 60, 2573-2577. [CrossRef]

31. Park, J.H.; Lee, Y.K.; Kim, K.M.; Kim, K.N. Bioactive calcium phosphate coating prepared on $\mathrm{H}_{2} \mathrm{O}_{2}$-treated titanium substrate by electrodeposition. Surf. Coat. Technol. 2005, 195, 252-257. [CrossRef]

32. Yang, X.; Zhang, B.; Lu, J.; Chen, J.; Zhang, X.; Gu, Z. Biomimetic Ca-P coating on pre-calcified Ti plates by electrodeposition method. Appl. Surf. Sci. 2010, 256, 2700-2704. [CrossRef]

33. Lin, D.Y.; Wang, X.X. Preparation of hydroxyapatite coating on smooth implant surface by electrodeposition. Ceram. Int. 2011, 37, 403-406. [CrossRef]

34. Mokabber, T.; Zhou, Q.; Vakis, A.I.; van Rijn, P.; Pei, Y.T. Mechanical and biological properties of electrodeposited calcium phosphate coatings. Mater. Sci. Eng. C 2019, 100, 475-484. [CrossRef] [PubMed]

35. Harun, W.S.W.; Asri, R.I.M.; Alias, J.; Zulkifli, F.H.; Kadirgama, K.; Ghani, S.A.C.; Shariffuddin, J.H.M. A comprehensive review of hydroxyapatite-based coatings adhesion on metallic biomaterials. Ceram. Int. 2018, 44, 1250-1268. [CrossRef]

36. Gopi, D.; Karthika, A.; Sekar, M.; Kavitha, L.; Pramod, R.; Dwivedi, J. Development of lotus-like hydroxyapatite coating on HELCDEB treated titanium by pulsed electrodeposition. Mater. Lett. 2013, 105, 216-219. [CrossRef]

37. Drevet, R.; Fauré, J.; Sayen, S.; Marle-Spiess, M.; El Btaouri, H.; Benhayoune, H. Electrodeposition of biphasic calcium phosphate coatings with improved dissolution properties. Mater. Chem. Phys. 2019, 236, 1-7. [CrossRef]

38. Fathyunes, L.; Khalil-Allafi, J. Effect of employing ultrasonic waves during pulse electrochemical deposition on the characteristics and biocompatibility of calcium phosphate coatings. Ultrason. Sonochem. 2018, 42, 293-302. [CrossRef]

39. Dudek, K.; Dulski, M.; Goryczka, T.; Gerle, A. Structural changes of hydroxyapatite coating electrophoretically deposited on NiTi shape memory alloy. Ceram. Int. 2018, 44, 11292-11300. [CrossRef]

40. Bartmanski, M.; Zielinski, A.; Majkowska-Marzec, B.; Strugala, G. Effects of solution composition and electrophoretic deposition voltage on various properties of nanohydroxyapatite coatings on the Ti13Zr13Nb alloy. Ceram. Int. 2018, 44, 19236-19246. [CrossRef]

41. Jażdżewska, M.; Majkowska-Marzec, B. Hydroxyapatite deposition on the laser modified Ti13Nb13Zr alloy. Adv. Mater. Sci. 2018, 17, 5-13. [CrossRef]

42. Patel, K.D.; Singh, R.K.; Lee, J.H.; Kim, H.W. Electrophoretic coatings of hydroxyapatite with various nanocrystal shapes. Mater. Lett. 2019, 234, 148-154. [CrossRef]

43. Araghi, A.; Hadianfard, M.J. Fabrication and characterization of functionally graded hydroxyapatite/TiO multilayer coating on Ti-6Al-4V titanium alloy for biomedical applications. Ceram. Int. 2015, 41, 12668-12679. [CrossRef]

44. Albayrak, O.; El-Atwani, O.; Altintas, S. Hydroxyapatite coating on titanium substrate by electrophoretic deposition method: Effects of titanium dioxide inner layer on adhesion strength and hydroxyapatite decomposition. Surf. Coat. Technol. 2008, 202, 2482-2487. [CrossRef]

45. Pavlović, M.R.P.; Eraković, S.G.; Pavlović, M.M.; Stevanović, J.S.; Panić, V.V.; Ignjatović, N.L. Anaphoretical/oxidative approach to the in-situ synthesis of adherent hydroxyapatite/titanium oxide composite coatings on titanium. Surf. Coat. Technol. 2019, 358, 688-694. [CrossRef]

46. Xiong, Y.; Lu, C.; Wang, C.; Song, R. Degradation behavior of n-MAO/EPD bio-ceramic composite coatings on magnesium alloy in simulated body fluid. J. Alloy. Compd. 2015, 625, 258-265. [CrossRef]

47. Fernando, N.L.; Kottegoda, N.; Jayanetti, S.; Karunaratne, V.; Jayasundara, D.R. Stability of nano-hydroxyapatite thin coatings at liquid/solid interface. Surf. Coat. Technol. 2018, 349, 24-31. [CrossRef]

48. Yu, J.M.; Choe, H.C. Mg-containing hydroxyapatite coatings on Ti-6Al-4V alloy for dental materials. Appl. Surf. Sci. 2018, 432, 294-299. [CrossRef]

49. Jeong, Y.H.; Kim, E.J.; Brantley, W.A.; Choe, H.C. Morphology of hydroxyapatite nanoparticles in coatings on nanotube-formed Ti-Nb-Zr alloys for dental implants. Vacuum 2014, 107, 297-303. [CrossRef] 
50. Bucur, A.I.; Linul, E.; Taranu, B.O. Hydroxyapatite coatings on Ti substrates by simultaneous precipitation and electrodeposition. Appl. Surf. Sci. 2020, 527, 146820. [CrossRef]

51. Sun, X.; Lin, H.; Zhang, C.; Jin, J.; Di, S. Electrochemical studies on CaP Electrodeposition on three dimensional surfaces of selective laser melted titanium scaffold. Coatings 2019, 9, 667. [CrossRef]

52. Parcharoen, Y.; Kajitvichyanukul, P.; Sirivisoot, S.; Termsuksawad, P. Hydroxyapatite electrodeposition on anodized titanium nanotubes for orthopedic applications. Appl. Surf. Sci. 2014, 311, 54-61. [CrossRef]

53. Vidal, E.; Buxadera-Palomero, J.; Pierre, C.; Manero, J.M.; Ginebra, M.P.; Cazalbou, S.; Combes, C.; Rupérez, E.; Rodríguez, D. Single-step pulsed electrodeposition of calcium phosphate coatings on titanium for drug delivery. Surf. Coat. Technol. 2019, 358, 266-275. [CrossRef]

54. Gopi, D.; Indira, J.; Kavitha, L. A comparative study on the direct and pulsed current electrodeposition of hydroxyapatite coatings on surgical grade stainless steel. Surf. Coat. Technol. 2012, 206, 2859-2869. [CrossRef]

55. Thanh, D.T.M.; Nam, P.T.; Phuong, N.T.; Que, L.X.; Van Anh, N.; Hoang, T.; Lam, T.D. Controlling the electrodeposition, morphology and structure of hydroxyapatite coating on 316L stainless steel. Mater. Sci. Eng. C 2013, 33, 2037-2045. [CrossRef] [PubMed]

56. Büyüksağiş, A.; Bulut, E.; Kayal1, Y. Corrosion behaviors of hydroxyapatite coated by electrodeposition method of Ti6Al4V, Ti and AISI 316L SS substrates. Prot. Met. Phys. Chem. Surf. 2013, 49, 776-787. [CrossRef]

57. Chakraborty, R.; Sengupta, S.; Saha, P.; Das, K.; Das, S. Synthesis of calcium hydrogen phosphate and hydroxyapatite coating on SS316 substrate through pulsed electrodeposition. Mater. Sci. Eng. C 2016, 69, 875-883. [CrossRef]

58. Chakraborty, R.; Saha, P. A comparative study on surface morphology and electrochemical behaviour of hydroacxyapatite-calcium hydrogen phosphate composite coating synthesized in-situ through electro chemical process under various deposition conditions. Surf. Interfaces 2018, 12, 160-167. [CrossRef]

59. Shibli, S.M.A.; Jayalekshmi, A.C. A novel nano hydroxyapatite-incorporated Ni-P coating as an effective inter layer for biological applications. J. Mater. Sci. Mater. Med. 2009, 20, 711-718. [CrossRef]

60. Lin, D.Y.; Wang, X.X. Electrodeposition of hydroxyapatite coating on CoNiCrMo substrate in dilute solution. Surf. Coat. Technol. 2010, 204, 3205-3213. [CrossRef]

61. Coşkun, M.I.; Karahan, I.H.; Yücel, Y. Optimized electrodeposition concentrations for hydroxyapatite coatings on CoCrMo biomedical alloys by computational techniques. Electrochim. Acta 2014, 150, 46-54. [CrossRef]

62. Etminanfar, M.R.; Khalil-Allafi, J.; Montaseri, A.; Vatankhah-Barenji, R. Endothelialization and the bioactivity of Ca-P coatings of different $\mathrm{Ca} / \mathrm{P}$ stoichiometry electrodeposited on the Nitinol superelastic alloy. Mater. Sci. Eng. C 2016, 62, 28-35. [CrossRef]

63. Etminanfar, M.R.; Khalil-Allafi, J.; Parsa, A.B. On the electrocrystallization of pure hydroxyapatite nanowalls on Nitinol alloy using a bipolar pulsed current. J. Alloy. Compd. 2016, 678, 549-555. [CrossRef]

64. Marashi-Najafi, F.; Khalil-Allafi, J.; Etminanfar, M.R. Biocompatibility of hydroxyapatite coatings deposited by pulse electrodeposition technique on the Nitinol superelastic alloy. Mater. Sci. Eng. C 2017, 76, 278-286. [CrossRef] [PubMed]

65. Sheykholeslami, S.O.R.; Khalil-Allafi, J.; Fathyunes, L. Preparation, characterization, and corrosion behavior of calcium phosphate coating electrodeposited on the modified nanoporous surface of NiTi alloy for biomedical applications. Metall. Mater. Trans. A Phys. Metall. Mater. Sci. 2018, 49, 5878-5887. [CrossRef]

66. Song, Y.W.; Shan, D.Y.; Han, E.H. Electrodeposition of hydroxyapatite coating on AZ91D magnesium alloy for biomaterial application. Mater. Lett. 2008, 62, 3276-3279. [CrossRef]

67. Zhang, C.Y.; Zeng, R.C.; Chen, R.S.; Liu, C.L.; Gao, J.C. Preparation of calcium phosphate coatings on Mg-1.0Ca alloy. Trans. Nonferrous Met. Soc. China Engl. Ed. 2010, 20, 655-659. [CrossRef]

68. Song, Y.; Zhang, S.; Li, J.; Zhao, C.; Zhang, X. Electrodeposition of Ca-P coatings on biodegradable Mg alloy: In vitro biomineralization behavior. Acta Biomater. 2010, 6, 1736-1742. [CrossRef]

69. Saremi, M.; Mohajernia, S.; Hejazi, S. Controlling the degradation rate of AZ31 Magnesium alloy and purity of nano-hydroxyapatit coating by pulse electrodeposition. Mater. Lett. 2014, 129, 111-113. [CrossRef]

70. Seyedraoufi, Z.S.; Mirdamadi, S. Effects of pulse electrodeposition parameters and alkali treatment on the properties of nano hydroxyapatite coating on porous MgeZn scaffold for bone tissue engineering application. Mater. Chem. Phys. 2014, 148, 519-527. [CrossRef] 
71. Seyedraoufi, Z.S.; Mirdamadi, S. In vitro biodegradability and biocompatibility of porous Mg-Zn scaffolds coated with nano hydroxyapatite via pulse electrodeposition. Trans. Nonferrous Met. Soc. China Engl. Ed. 2015, 25, 4018-4027. [CrossRef]

72. Monasterio, N.; Ledesma, J.L.; Aranguiz, I.; Garcia-Romero, A.; Zuza, E. Analysis of electrodeposition processes to obtain calcium phosphate layer on AZ31 alloy. Surf. Coat. Technol. 2017, 319, 12-22. [CrossRef]

73. Han, J.; Blawert, C.; Tang, S.; Yang, J.; Hu, J.; Zheludkevich, M.L. Effect of surface pre-treatments on the formation and degradation behaviour of a calcium phosphate coating on pure magnesium. Coatings 2019, 9, 259. [CrossRef]

74. Liao, Y.M.; De Feng, Z.; Li, S.W. Preparation and characterization of hydroxyapatite coatings on human enamel by electrodeposition. Thin Solid Film. 2008, 516, 6145-6150. [CrossRef]

75. Benea, L.; Danaila, E.; Ponthiaux, P. Effect of titania anodic formation and hydroxyapatite electrodeposition on electrochemical behaviour of Ti-6Al-4V alloy under fretting conditions for biomedical applications. Corros. Sci. 2015, 91, 262-271. [CrossRef]

76. Liu, S.; Li, H.; Zhang, L.; Yin, X.; Guo, Y. In simulated body fluid performance of polymorphic apatite coatings synthesized by pulsed electrodeposition. Mater. Sci. Eng. C 2017, 79, 100-107. [CrossRef] [PubMed]

77. Zhao, X.; Chen, X.; Zhang, L.; Liu, Q.; Wang, Y.; Zhang, W.; Zheng, J. Preparation of nano-hydroxyapatite coated carbon nanotube reinforced hydroxyapatite composites. Coatings 2018, 8, 357. [CrossRef]

78. Li, T.T.; Ling, L.; Lin, M.C.; Jiang, Q.; Lin, Q.; Lou, C.W.; Lin, J.H. Effects of ultrasonic treatment and current density on the properties of hydroxyapatite coating via electrodeposition and its in vitro biomineralization behavior. Mater. Sci. Eng. C 2019, 105, 110062. [CrossRef] [PubMed]

79. Gopi, D.; Karthika, A.; Nithiya, S.; Kavitha, L. In vitro biological performance of minerals substituted hydroxyapatite coating by pulsed electrodeposition method. Mater. Chem. Phys. 2014, 144, 75-85. [CrossRef]

80. Drevet, R.; Benhayoune, H. Pulsed electrodeposition for the synthesis of strontium-substituted calcium phosphate coatings with improved dissolution properties. Mater. Sci. Eng. C 2013, 33, 4260-4265. [CrossRef]

81. Yajing, Y.; Qiongqiong, D.; Yong, H.; Han, S.; Pang, X. Magnesium substituted hydroxyapatite coating on titanium with nanotublar $\mathrm{TiO}_{2}$ intermediate layer via electrochemical deposition. Appl. Surf. Sci. 2014, 305, 77-85. [CrossRef]

82. Huang, Y.; Hao, M.; Nian, X.; Qiao, H.; Zhang, X.X.; Zhang, X.X.; Song, G.; Guo, J.; Pang, X.; Zhang, H. Strontium and copper co-substituted hydroxyapatite-based coatings with improved antibacterial activity and cytocompatibility fabricated by electrodeposition. Ceram. Int. 2016, 42, 11876-11888. [CrossRef]

83. Huang, Y.; Qiao, H.; Nian, X.; Zhang, X.; Zhang, X.; Song, G.; Xu, Z.; Zhang, H.; Han, S. Improving the bioactivity and corrosion resistance properties of electrodeposited hydroxyapatite coating by dual doping of bivalent strontium and manganese ion. Surf. Coat. Technol. 2016, 291, 205-215. [CrossRef]

84. Fu, D.L.; Jiang, Q.H.; He, F.M.; Fu, B.P. Adhesion of bone marrow mesenchymal stem cells on porous titanium surfaces with strontium-doped hydroxyapatite coating. J. Zhejiang Univ. Sci. B 2017, 18, 778-788. [CrossRef]

85. Huang, Y.; Wang, W.; Zhang, X.; Liu, X.; Xu, Z.; Han, S.; Su, Z.; Liu, H.; Gao, Y.; Yang, H. A prospective material for orthopedic applications: Ti substrates coated with a composite coating of a titania-nanotubes layer and a silver-manganese-doped hydroxyapatite layer. Ceram. Int. 2017, 44, 5528-5542. [CrossRef]

86. Karthika, A. Aliovalent ions substituted hydroxyapatite coating on titanium for improved medical applications. Mater. Today Proc. 2018, 5, 8768-8774. [CrossRef]

87. Drevet, R.; Zhukova, Y.; Dubinskiy, S.; Kazakbiev, A.; Naumenko, V.; Abakumov, M.; Fauré, J.; Benhayoune, H.; Prokoshkin, S. Electrodeposition of cobalt-substituted calcium phosphate coatings on Ti22Nb6Zr alloy for bone implant applications. J. Alloys Compd. 2019, 793, 576-582. [CrossRef]

88. Furko, M.; Della Bella, E.; Fini, M.; Balázsi, C. Corrosion and biocompatibility examination of multi-element modified calcium phosphate bioceramic layers. Mater. Sci. Eng. C 2019, 95, 381-388. [CrossRef]

89. Meng, E.C.; Guan, S.K.; Wang, H.X.; Wang, L.G.; Zhu, S.J.; Hu, J.H.; Ren, C.X.; Gao, J.H.; Feng, Y.S. Effect of electrodeposition modes on surface characteristics and corrosion properties of fluorine-doped hydroxyapatite coatings on Mg-Zn-Ca alloy. Appl. Surf. Sci. 2011, 257, 4811-4816. [CrossRef]

90. Qiu, X.; Wan, P.; Tan, L.; Fan, X.; Yang, K. Preliminary research on a novel bioactive silicon doped calcium phosphate coating on AZ31 magnesium alloy via electrodeposition. Mater. Sci. Eng. C 2014, 36, 65-76. [CrossRef]

91. Aboudzadeh, N.; Dehghanian, C.; Shokrgozar, M.A. Effect of electrodeposition parameters and substrate on morphology of Si-HA coating. Surf. Coat. Technol. 2019, 375, 341-351. [CrossRef] 
92. Liu, S.J.; Li, H.J.; Zhang, L.L.; Feng, L.; Yao, P. Strontium and magnesium substituted dicalcium phosphate dehydrate coating for carbon/carbon composites prepared by pulsed electrodeposition. Appl. Surf. Sci. 2015, 359, 288-292. [CrossRef]

93. Peñaflor Galindo, T.G.; Kataoka, T.; Fujii, S.; Okuda, M.; Tagaya, M. Preparation of nanocrystalline zinc-substituted hydroxyapatite films and their biological properties. Colloids Interface Sci. Commun. 2016, 10-11, 15-19. [CrossRef]

94. Huang, C.N.; Tang, Z.T.; Chan, F.E.; Burnouf, T.; Huang, W.C.; Chen, P.C. Fabrication of co-electrodeposition of plasma proteins/iridium oxide hybrid films. Ceram. Int. 2018, 44, 117-120. [CrossRef]

95. Gao, X.; Gui, R.; Guo, H.; Wang, Z.; Liu, Q. Creatinine-induced specific signal responses and enzymeless ratiometric electrochemical detection based on copper nanoparticles electrodeposited on reduced graphene oxide-based hybrids. Sens. Actuators B Chem. 2019, 285, 201-208. [CrossRef]

96. Benea, L.; Celis, J.P. Reactivity of porous titanium oxide film and chitosan layer electrochemically formed on Ti-6Al-4V alloy in biological solution. Surf. Coat. Technol. 2018, 354, 145-152. [CrossRef]

97. Nakano, K.; Egashira, K.; Masuda, S.; Funakoshi, K.; Zhao, G.; Kimura, S.; Matoba, T.; Sueishi, K.; Endo, Y.; Kawashima, Y.; et al. Formulation of nanoparticle-eluting stents by a cationic electrodeposition coating technology. efficient nano-drug delivery via bioabsorbable polymeric nanoparticle-eluting stents in porcine coronary arteries. JACC Cardiovasc. Interv. 2009, 2, 277-283. [CrossRef]

98. Jiang, P.L.; Hou, R.Q.; Chen, C.D.; Sun, L.; Dong, S.G.; Pan, J.S.; Lin, C.J. Controllable degradation of medical magnesium by electrodeposited composite films of mussel adhesive protein (Mefp-1) and chitosan. J. Colloid Interface Sci. 2016, 478, 246-255. [CrossRef]

99. Meng, L.; Li, Y.; Pan, K.; Zhu, Y.; Wei, W.; Li, X.; Liu, X. Colloidal particle based electrodeposition coatings on NiTi alloy: Reduced releasing of nickel ions and improved biocompatibility. Mater. Lett. 2018, 230, $228-231$. [CrossRef]

100. Sak, A.; Moskalewicz, T.; Zimowski, S.; Cieniek, Ł.; Dubiel, B.; Radziszewska, A.; Kot, M.; Łukaszczyk, A. Influence of polyetheretherketone coatings on the Ti-13Nb-13Zr titanium alloy's bio-tribological properties and corrosion resistance. Mater. Sci. Eng. C 2016, 63, 52-61. [CrossRef]

101. Chakraborty, R.; Seesala, V.S.; Sengupta, S.; Dhara, S.; Saha, P.; Das, K.; Das, S. Comparison of Osteoconduction, cytocompatibility acand corrosion protection performance of hydroxyapatite-calcium hydrogen phosphate composite coating synthesized in-situ through pulsed electro-deposition with varying amount of phase and crystallinity. Surf. Interfaces 2018, 10, 1-10. [CrossRef]

102. Shojaee, P.; Afshar, A. Effects of zirconia content on characteristics and corrosion behavior of hydroxyapatite/ZrO2 biocomposite coatings codeposited by electrodeposition. Surf. Coat. Technol. 2015, 262, 166-172. [CrossRef]

103. Chakraborty, R.; Seesala, V.S.; Sen, M.; Sengupta, S.; Dhara, S.; Saha, P.; Das, K.; Das, S. MWCNT reinforced bone like calcium phosphate-Hydroxyapatite composite coating developed through pulsed electrodeposition with varying amount of apatite phase and crystallinity to promote superior osteoconduction, cytocompatibility and corrosion protection. Surf. Coat. Technol. 2017, 325, 496-514. [CrossRef]

104. Huang, Y.; Han, S.; Pang, X.; Ding, Q.; Yan, Y. Electrodeposition of porous hydroxyapatite/calcium silicate composite coating on titanium for biomedical applications. Appl. Surf. Sci. 2013, 271, 299-302. [CrossRef]

105. Huang, Y.; Zhang, H.; Qiao, H.; Nian, X.; Zhang, X.; Wang, W.; Zhang, X.; Chang, X.; Han, S.; Pang, X. Anticorrosive effects and in vitro cytocompatibility of calcium silicate/zinc-doped hydroxyapatite composite coatings on titanium. Appl. Surf. Sci. 2015, 357, 1776-1784. [CrossRef]

106. Mostafa, N.Y.; Montaser, A.; Al-Affray, R.A.; Kamel, M.M.; Alhadhrami, A. Processing and characterization of novel calcium titanate/Na-titanate nanotube/rutile nanocomposite coating on titanium metal. Appl. Phys. A Mater. Sci. Process. 2019, 125, 1-6. [CrossRef]

107. Chozhanathmisra, M.; Murugan, N.; Karthikeyan, P.; Sathishkumar, S.; Anbarasu, G.; Rajavel, R. Development of antibacterial activity and corrosion resistance properties of electrodeposition of mineralized hydroxyapatite coated on titanium alloy for biomedical applications. Mater. Today Proc. 2017, 4, 12393-12400. [CrossRef]

108. Chozhanathmisra, M.; Ramya, S.; Kavitha, L.; Gopi, D. Development of zinc-halloysite nanotube/minerals substituted hydroxyapatite bilayer coatings on titanium alloy for orthopedic applications. Colloids Surf. A Physicochem. Eng. Asp. 2016, 511, 357-365. [CrossRef]

109. Fathyunes, L.; Khalil-Allafi, J. Characterization and corrosion behavior of graphene oxide-hydroxyapatite composite coating applied by ultrasound-assisted pulse electrodeposition. Ceram. Int. 2017, 43, 13885-13894. [CrossRef] 
110. Fathyunes, L.; Khalil-Allafi, J.; Sheykholeslami, S.O.R.; Moosavifar, M. Biocompatibility assessment of graphene oxide-hydroxyapatite coating applied acon $\mathrm{TiO}_{2}$ nanotubes by ultrasound-assisted pulse electrodeposition. Mater. Sci. Eng. C 2018, 87, 10-21. [CrossRef]

111. Zhang, L.; Zhu, F.; Li, H.; Zhao, F.; Li, S. A duplex coating composed of electrophoretic deposited graphene oxide inner-layer and electrodeposited graphene oxide/Mg substituted hydroxyapatite outer-layer on carbon/carbon composites for biomedical application. Ceram. Int. 2018, 44, 21229-21237. [CrossRef]

112. Gopi, D.; Shinyjoy, E.; Sekar, M.; Surendiran, M.; Kavitha, L.; Sampath Kumar, T.S. Development of carbon nanotubes reinforced hydroxyapatite composite coatings on titanium by electrodeposition method. Corros. Sci. 2013, 73, 321-330. [CrossRef]

113. Fathyunes, L.; Khalil-Allafi, J.; Moosavifar, M. Development of graphene oxide/calcium phosphate coating by pulse electrodeposition on anodized titanium: Biocorrosion and mechanical behaavior. J. Mech. Behav. Biomed. Mater. 2019, 90, 575-586. [CrossRef] [PubMed]

114. Khazeni, D.; Saremi, M.; Soltani, R. Development of HA-CNTs composite coating on AZ31 magnesium alloy by cathodic electrodeposition. Part 1: Microstructural and mechanical characterization. Ceram. Int. 2019, 45, 11174-11185. [CrossRef]

115. Chakraborty, R.; Manna, J.S.; Das, D.; Sen, M.; Saha, P. A comparative outlook of corrosion behaviour and chlorophyll assisted growth kinetics of various carbon nano-structure reinforced hydroxyapatite-calcium orthophosphate coating synthesized in-situ through pulsed electrochemical deposition. Appl. Surf. Sci. 2019, 475, 28-42. [CrossRef]

116. Długoń, E.; Niemiec, W.; Frączek-Szczypta, A.; Jeleń, P.; Sitarz, M.; Błazewicz, M. Spectroscopic studies of electrophoretically deposited hybrid HAp/CNT coatings on titanium. Spectrochim. Acta Part. A Mol. Biomol. Spectrosc. 2014, 133, 872-875. [CrossRef]

117. Maleki-Ghaleh, H.; Khalil-Allafi, J. Characterization, mechanical and in vitro biological behavior of hydroxyapatite-titanium-carbon nanotube composite coatings deposited on NiTi alloy by electrophoretic deposition. Surf. Coat. Technol. 2019, 363, 179-190. [CrossRef]

118. Poorraeisi, M.; Afshar, A. The study of electrodeposition of hydroxyapatite- $\mathrm{ZrO}_{2}-\mathrm{TiO}_{2}$ nanocomposite coatings on 316 stainless steel. Surf. Coat. Technol. 2018, 339, 199-207. [CrossRef]

119. Pei, L.; Zhang, B.; Luo, H.; Wu, X.; Li, G.; Sheng, H.; Zhang, L. Electrodeposition of ZnO Nanoprism-Zn substituted hydroxyapatite duplex layer coating for carbon fiber. Ceram. Int. 2019, 45, 14278-14286. [CrossRef]

120. Göncü, Y.; Geçgin, M.; Bakan, F.; Ay, N. Electrophoretic deposition of hydroxyapatite-hexagonal boron nitride composite coatings on Ti substrate. Mater. Sci. Eng. C 2017, 79, 343-353. [CrossRef]

121. Khanmohammadi, S.; Ilkhchi, M.O.; Khalil-Allafi, J. Electrophoretic deposition and characterization of bioglass-whisker hydroxyapatite nanocomposite coatings on titanium substrate. Surf. Coat. Technol. 2019, 378, 124949. [CrossRef]

122. Yılmaz, E.; Çakıroğlu, B.; Gökçe, A.; Findik, F.; Gulsoy, H.O.; Gulsoy, N.; Mutlu, Ö.; Özacar, M. Novel hydroxyapatite/graphene oxide/collagen bioactive composite coating on Ti16Nb alloys by electrodeposition. Mater. Sci. Eng. C 2019, 101, 292-305. [CrossRef]

123. Kumar, A.M.; Adesina, A.Y.; Hussein, M.A.; Ramakrishna, S.; Al-Aqeeli, N.; Akhtar, S.; Saravanan, S. PEDOT/FHA nanocomposite coatings on newly developed Ti-Nb-Zr implants: Biocompatibility and surface protection against corrosion and bacterial infections. Mater. Sci. Eng. C 2019, 98, 482-495. [CrossRef] [PubMed]

124. Chakraborty, R.; Seesala, V.S.; Manna, J.S.; Saha, P.; Dhara, S. Synthesis, characterization and cytocompatibility assessment of hydroxyapatite-polypyrrole composite coating synthesized through pulsed reverse electrochemical deposition. Mater. Sci. Eng. C 2019, 94, 597-607. [CrossRef] [PubMed]

125. Pang, X.; Zhitomirsky, I. Electrodeposition of hydroxyapatite-silver-chitosan nanocomposite coatings. Surf. Coat. Technol. 2008, 202, 3815-3821. [CrossRef]

126. Yan, Y.; Zhang, X.; Li, C.; Huang, Y.; Ding, Q.; Pang, X. Preparation and characterization of chitosan-silver/ hydroxyapatite composite coatings onTiO $\mathrm{T}_{2}$ nanotube for biomedical applications. Appl. Surf. Sci. 2015, 332, 62-69. [CrossRef]

127. Zhao, P.; Zhao, Y.; Xiao, L.; Deng, H.; Du, Y.; Chen, Y.; Shi, X. Electrodeposition to construct free-standing chitosan/layered double hydroxides hydro-membrane for electrically triggered protein release. Colloids Surf. B Biointerfaces 2017, 158, 474-479. [CrossRef] 
128. Zhong, Z.; Qin, J.; Ma, J. Electrophoretic deposition of biomimetic zinc substituted hydroxyapatite coatings with chitosan and carbon nanotubes on titanium. Ceram. Int. 2015, 41, 8878-8884. [CrossRef]

129. Clifford, A.; Lee, B.E.J.; Grandfield, K.; Zhitomirsky, I. Biomimetic modification of poly-L-lysine and electrodeposition of nanocomposite coatings for orthopaedic applications. Colloids Surf. B Biointerfaces 2019, 176, 115-121. [CrossRef]

130. Baştan, F.E.; Atiq Ur Rehman, M.; Avcu, Y.Y.; Avcu, E.; Üstel, F.; Boccaccini, A.R. Electrophoretic co-deposition of PEEK-hydroxyapatite composite coatings for biomedical applications. Colloids Surf. B Biointerfaces 2018, 169, 176-182. [CrossRef]

131. Patiño-Herrera, R.; González-Alatorre, G.; Estrada-Baltazar, A.; Escoto-Chavéz, S.E.; Pérez, E. Hydrophobic coatings for prevention of dental enamel erosion. Surf. Coat. Technol. 2015, 275, 148-154. [CrossRef]

132. Khabazian, S.; Sanjabi, S. The effect of multi-walled carbon nanotube pretreatments on the electrodeposition of Ni-MWCNTs coatings. Appl. Surf. Sci. 2011, 257, 5850-5856. [CrossRef]

133. El-Wassefy, N.A.; Reicha, F.M.; Aref, N.S. Electro-chemical deposition of nano hydroxyapatite-zinc coating on titanium metal substrate. Int. J. Implant. Dent. 2017, 3, 1-8. [CrossRef] [PubMed]

134. Fu, C.; Zhang, X.; Savino, K.; Gabrys, P.; Gao, Y.; Chaimayo, W.; Miller, B.L.; Yates, M.Z. Antimicrobial silver-hydroxyapatite composite coatings through two-stage electrochemical synthesis. Surf. Coat. Technol. 2016, 301, 13-19. [CrossRef]

135. Bartmanski, M.; Cieslik, B.; Glodowska, J.; Kalka, P.; Pawlowski, L.; Pieper, M.; Zielinski, A. Electrophoretic deposition (EPD) of nanohydroxyapatite-nanosilver coatings on Ti13Zr13Nb alloy. Ceram. Int. 2017, 43, 11820-11829. [CrossRef]

136. Bartmanski, M. The properties of nanosilver-Doped nanohydroxyapatite coating on the Ti13zr13Nb alloy. Adv. Mater. Sci. 2017, 17, 18-28. [CrossRef]

137. Bartmanski, M.; Zielinski, A.; Jazdzewska, M.; Głodowska, J.; Kalka, P. Effects of electrophoretic deposition times and nanotubular oxide surfaces on properties of the nanohydroxyapatite/nanocopper coating on the Ti13Zr13Nb alloy. Ceram. Int. 2019, 45, 20002-20010. [CrossRef]

138. Majkowska-Marzec, B.; Rogala-Wielgus, D.; Bartmański, M.; Bartosewicz, B.; Zieliński, A.S. Comparison of Properties of the Hybrid and Bilayer MWCNTs-Hydroxyapatite Coatings on Ti Alloy. Coatings 2019, 9, 643. [CrossRef]

139. Jang, J.M.; Kim, S.D.; Park, T.E.; Choe, H.C. Ultra-fine structures of Pd-Ag-HAp nanoparticle deposition on protruded $\mathrm{TiO}_{2}$ barrier layer for dental implant. Appl. Surf. Sci. 2018, 432, 285-293. [CrossRef]

140. Silva-Ichante, M.; Reyes-Vidal, Y.; Bácame-Valenzuela, F.J.; Ballesteros, J.C.; Arciga, E.; Ţălu, Ş.; Méndez-Albores, A.; Trejo, G. Electrodeposition of antibacterial $\mathrm{Zn}-\mathrm{Cu}$ /silver nanoparticle (AgNP) composite coatings from an alkaline solution containing glycine and AgNPs. J. Electroanal. Chem. 2018, 823, 328-334. [CrossRef]

141. Kumar, A.M.; Nagarajan, S.; Ramakrishna, S.; Sudhagar, P.; Kang, Y.S.; Kim, H.; Gasem, Z.M.; Rajendran, N. Electrochemical and in vitro bioactivity of polypyrrole/ceramic nanocomposite coatings on 316L SS bio-implants. Mater. Sci. Eng. C 2014, 43, 76-85. [CrossRef]

142. Lázaro-Martínez, J.M.; Byrne, A.J.; Rodríguez-Castellón, E.; Manrique, J.M.; Jones, L.R.; Dall'Orto, V.C. Linear polyethylenimine-decorated gold nanoparticles: One-step electrodeposition and studies of interaction with viral and animal proteins. Electrochim. Acta 2019, 301, 126-135. [CrossRef]

143. Wang, Z.; Zhang, X.; Gu, J.; Yang, H.; Nie, J.; Ma, G. Electrodeposition of alginate/chitosan layer-by-layer composite coatings on titanium substrates. Carbohydr. Polym. 2014, 103, 38-45. [CrossRef] [PubMed]

144. Kumar, A.M.; Suresh, B.; Das, S.; Obot, I.B.; Adesina, A.Y.; Ramakrishna, S. Promising bio-composites of polypyrrole and chitosan: Surface protective and in vitro biocompatibility performance on 316L SS implants. Carbohydr. Polym. 2017, 173, 121-130. [CrossRef] [PubMed]

145. Pawłowski, Ł.; Bartmański, M.; Strugała, G.; Mielewczyk-Gryń, A.; Jażdżewska, M.; Zieliński, A. Electrophoretic deposition and characterization of Chitosan/Eudragit E 100 coatings on titanium substrate. Coatings 2020, 10, 607. [CrossRef]

146. Bonifacio, M.A.; Cometa, S.; Dicarlo, M.; Baruzzi, F.; de Candia, S.; Gloria, A.; Giangregorio, M.M.; Mattioli-Belmonte, M.; De Giglio, E. Gallium-modified chitosan/poly(acrylic acid) bilayer coatings for improved titanium implant performances. Carbohydr. Polym. 2017, 166, 348-357. [CrossRef] 
147. Trzaskowska, P.A.; Poniatowska, A.; Tokarska, K.; Wiśniewski, C.; Ciach, T.; Malinowska, E. Promising electrodeposited biocompatible coatings for steel obtained from polymerized microemulsions. Colloids Surf. A Physicochem. Eng. Asp. 2020, 591, 124555. [CrossRef]

148. Liu, L.; Qi, W.; Gao, X.; Wang, C.; Wang, G. Synergistic effect of metal ion additives on graphitic carbon nitride nanosheet-templated electrodeposition of $\mathrm{Cu} @ \mathrm{CuO}$ for enzyme-free glucose detection. J. Alloys Compd. 2018, 745, 155-163. [CrossRef]

149. Zhao, Z.W.; Zhang, G.; Li, H.G. Preparation of calcium phosphate coating on pure titanium substrate by electrodeposition method. J. Cent. South. Univ. Technol. Engl. Ed. 2004, 11, 147-151. [CrossRef]

150. Hou, X.; Liu, X.; Xu, J.; Shen, J.; Liu, X. A self-optimizing electrodeposition process for fabrication of calcium phosphate coatings. Mater. Lett. 2001, 50, 103-107. [CrossRef]

151. Manso, M.; Jiménez, C.; Morant, C.; Herrero, P.; Martínez-Duart, J. Electrodeposition of hydroxyapatite coatings in basic conditions. Biomaterials 2000, 21, 1755-1761. [CrossRef]

152. Beaufils, S.; Rouillon, T.; Millet, P.; Le Bideau, J.; Weiss, P.; Chopart, J.P.; Daltin, A.L. Synthesis of calcium-deficient hydroxyapatite nanowires and nanotubes performed by template-assisted electrodeposition. Mater. Sci. Eng. C 2019, 98, 333-346. [CrossRef]

153. Khalili, V.; Khalil-Allafi, J.; Frenzel, J.; Eggeler, G. Bioactivity and electrochemical behavior of hydroxyapatite-silicon-multi walled carbon nano-tubes composite coatings synthesized by EPD on NiTi alloys in simulated body fluid. Mater. Sci. Eng. C 2017, 71, 473-482. [CrossRef] [PubMed]

154. Pawlik, A.; Rehman, M.A.U.; Nawaz, Q.; Bastan, F.E.; Sulka, G.D.; Boccaccini, A.R. Fabrication and characterization of electrophoretically deposited chitosan-hydroxyapatite composite coatings on anodic titanium dioxide layers. Electrochim. Acta 2019, 307, 465-473. [CrossRef]

155. Molaei, A.; Yari, M.; Afshar, M.R. Modification of electrophoretic deposition of chitosan-bioactive glass-hydroxyapatite nanocomposite coatings for orthopedic applications by changing voltage and deposition time. Ceram. Int. 2015, 41, 14537-14544. [CrossRef]

156. Durdu, S.; Usta, M.; Berkem, A.S. Bioactive coatings on Ti6Al4V alloy formed by plasma electrolytic oxidation. Surf. Coat. Technol. 2016, 301, 85-93. [CrossRef]

157. Liu, S.; Li, B.; Liang, C.; Wang, H.; Qiao, Z. Formation mechanism and adhesive strength of a hydroxyapatite/ $\mathrm{TiO}_{2}$ composite coating on a titanium surface prepared by micro-arc oxidation. Appl. Surf. Sci. 2016, 362, 109-114. [CrossRef]

158. Shi, M.; Li, H. The morphology, structure and composition of microarc oxidation (MAO) ceramic coating in Ca-P electrolyte with complexing agent EDTMPS and interpretation hypothesis of MAO process. Surf. Eng. Appl. Electrochem. 2016, 52, 32-42. [CrossRef]

159. Mao, Y.; Yan, J.; Wang, L.; Dong, W.; Jia, Y.; Hu, X.; Wang, X. Formation and properties of bioactive barium titanate coatings produced by plasma electrolytic oxidation. Ceram. Int. 2018, 44, 12978-12986. [CrossRef]

160. Luo, S.; Wang, Q.; Ye, R.; Ramachandran, C.S. Effects of electrolyte concentration on the microstructure and properties of plasma electrolytic oxidation coatings on Ti-6Al-4V alloy. Surf. Coat. Technol. 2019, 375, 864-876. [CrossRef]

161. Zhang, X.; Yu, Y.; Jiang, D.; Jiao, Y.; Wu, Y.; Peng, Z.; Zhou, J.; Wu, J.; Dong, Z. Synthesis and characterization of a bi-functional hydroxyapatite/Cu-doped $\mathrm{TiO}_{2}$ composite coating. Ceram. Int. 2019, 45, 6693-6701. [CrossRef]

162. Dziaduszewska, M.; Shimabukuro, M.; Seramak, T.; Zielinski, A. Effects of micro-arc oxidation process parameters on characteristics of calcium-phosphate containing oxide layers on the selective laser melted Ti13Zr13Nb alloy. Coatings 2020, 10, 745. [CrossRef]

163. Antonio, R.F.; Rangel, E.C.; Mas, B.A.; Duek, E.A.R.; Cruz, N.C. Growth of hydroxyapatite coatings on tantalum by plasma electrolytic oxidation in a single step. Surf. Coat. Technol. 2019, 357, 698-705. [CrossRef]

164. Cengiz, S.; Azakli, Y.; Tarakci, M.; Stanciu, L.; Gencel, Y. Microarc oxidation type discharge types and bio properties of the coating synthesized on zirconium. Mater. Sci. Eng. C. 2017, 77, 374-383. [CrossRef] [PubMed]

165. Kim, Y.K.; Jang, Y.S.; Kim, S.Y.; Lee, M.H. Functions achieved by the hyaluronic acid derivatives coating and hydroxide film on bio-absorbed Mg. Appl. Surf. Sci. 2019, 473, 31-39. [CrossRef]

166. Durdu, S.; Aktuğ, S.L.; Korkmaz, K. Characterization and mechanical properties of the duplex coatings produced on steel by electro-spark deposition and micro-arc oxidation. Surf. Coat. Technol. 2013, 236, 303-308. [CrossRef] 
167. Durdu, S.; Korkmaz, K.; Aktuğ, S.L.; Çakır, A. Characterization and bioactivity of hydroxyapatite-based coatings formed on steel by electro-spark deposition and micro-arc oxidation. Surf. Coat. Technol. 2017, 326, 111-120. [CrossRef]

168. Aliyu, A.A.; Abdul-Rani, A.M.; Rao, T.V.V.L.N.; Axinte, E.; Hastuty, S.; Parameswari, R.P.; Subramaniam, J.R.; Thyagarajan, S.P. Characterization, adhesion strength and in-vitro cytotoxicity investigation of hydroxyapatite coating synthesized on Zr-based BMG by electro discharge process. Surf. Coat. Technol. 2019, 370, $213-226$. [CrossRef]

169. Devgan, S.; Sidhu, S.S. Surface modification of $\beta$-type titanium with multi-walled CNTs/ $\mu$-HAp powder mixed electro discharge treatment process. Mater. Chem. Phys. 2020, 239, 122005. [CrossRef]

170. Momeni, M.M.; Nazari, Z. Pt/PANI-MWCNTs nanocomposite coating prepared by electropolymerisationelectrodeposition for glycerol electro-oxidation. Surf. Eng. 2015, 31, 472-479. [CrossRef]

171. Rikhari, B.; Mani, S.P.; Rajendran, N. Electrochemical behavior of polypyrrole/chitosan composite coating on Ti metal for biomedical applications. Carbohydr. Polym. 2018, 189, 126-137. [CrossRef]

172. Almeida, L.C.; Correia, R.D.; Squillaci, G.; Morana, A.; La Cara, F.; Correia, J.P.; Viana, A.S. Electrochemical deposition of bio-inspired laccase-polydopamine films for phenolic sensors. Electrochim. Acta 2019, 319, 462-471. [CrossRef]

(C) 2020 by the authors. Licensee MDPI, Basel, Switzerland. This article is an open access article distributed under the terms and conditions of the Creative Commons Attribution (CC BY) license (http://creativecommons.org/licenses/by/4.0/). 